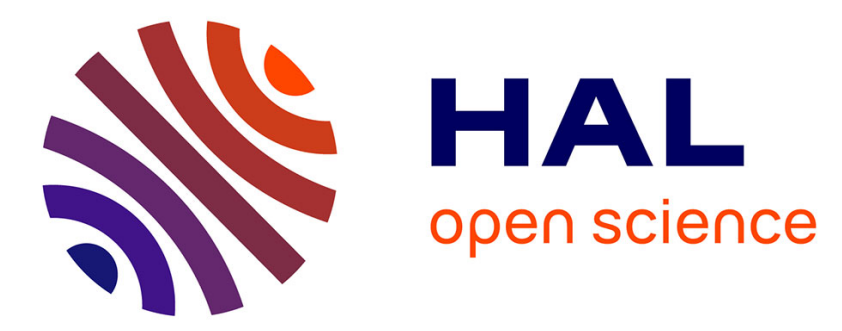

\title{
Bifurcation Routes to Volatility Clustering under Evolutionary Learning
}

Andrea Gaunersdorfer, Cars H. Hommes, Florian O.O. Wagener

\section{To cite this version:}

Andrea Gaunersdorfer, Cars H. Hommes, Florian O.O. Wagener. Bifurcation Routes to Volatility Clustering under Evolutionary Learning. Journal of Economic Behavior and Organization, 2008, 67 (1), pp.27. 10.1016/j.jebo.2007.07.004 . hal-00598267

\section{HAL Id: hal-00598267 https://hal.science/hal-00598267}

Submitted on 6 Jun 2011

HAL is a multi-disciplinary open access archive for the deposit and dissemination of scientific research documents, whether they are published or not. The documents may come from teaching and research institutions in France or abroad, or from public or private research centers.
L'archive ouverte pluridisciplinaire HAL, est destinée au dépôt et à la diffusion de documents scientifiques de niveau recherche, publiés ou non, émanant des établissements d'enseignement et de recherche français ou étrangers, des laboratoires publics ou privés. 


\section{Accepted Manuscript}

Title: Bifurcation Routes to Volatility Clustering under Evolutionary Learning

Authors: Andrea Gaunersdorfer, Cars H. Hommes, Florian O.O. Wagener

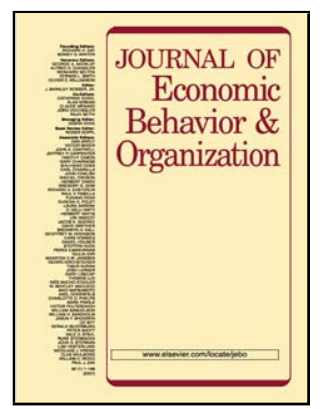

PII:

S0167-2681(07)00158-8

DOI: doi:10.1016/j.jebo.2007.07.004

Reference: JEBO 2130

To appear in: Journal of Economic Behavior \& Organization

Received date: 6-12-2006

Revised date: $\quad 11-7-2007$

Accepted date: $\quad 13-7-2007$

Please cite this article as: Gaunersdorfer, A., Hommes, C.H., Wagener, F.O.O., Bifurcation Routes to Volatility Clustering under Evolutionary Learning, Journal of Economic Behavior and Organization (2007), doi:10.1016/j.jebo.2007.07.004

This is a PDF file of an unedited manuscript that has been accepted for publication. As a service to our customers we are providing this early version of the manuscript. The manuscript will undergo copyediting, typesetting, and review of the resulting proof before it is published in its final form. Please note that during the production process errors may be discovered which could affect the content, and all legal disclaimers that apply to the journal pertain. 


\title{
Bifurcation Routes to Volatility Clustering under Evolutionary Learning
}

\author{
Andrea Gaunersdorfer \\ University of Vienna, Department of Finance \\ Brünner Straße 72, 1210 Wien, Austria \\ Cars H. Hommes \\ Florian O. O. Wagener \\ Center for Nonlinear Dynamics in Economics and Finance, University of Amsterdam \\ Roetersstraat 11, 1018 WB Amsterdam, The Netherlands \\ Revised draft, July 9, 2007

\begin{abstract}
A simple asset pricing model with two types of boundedly rational traders, fundamentalists and chartists, is studied. Fractions of trader types change over time according to evolutionary learning, with chartists conditioning their forecasting rule upon deviations from a benchmark fundamental. Volatility clustering arises endogenously and two generic mechanisms are proposed as an explanation: (1) coexistence of a stable steady state and a stable limit cycle, due to a so-called Chenciner bifurcation of the system, and (2) intermittency and associated bifurcation routes to strange attractors. Economic intuition as to why these phenomena arise in nonlinear multi-agent evolutionary systems is provided.
\end{abstract}

JEL classification: E32, G12, D84

Keywords: multi-agent systems, bounded rationality, evolutionary learning, bifurcations and chaos, coexisting attractors

Acknowledgments. An earlier draft of this paper has been presented at the SCE-meeting at Yale, June 28-29, 2001, at the workshop on Economic Dynamics at the University of Amsterdam, January 4-6, 2001, and at various seminars. We would like to thank all participants for stimulating discussions. Special thanks are due to Buz Brock for extensive discussions and detailed comments on an earlier draft. We are also grateful to Jess Benhabib, Barkley Rosser and an anonymous referee for their encouragement and comments. This research was supported by the Austrian Science Foundation (FWF) under grant SFB\#010 ('Adaptive Information Systems and Modeling in Economics and Management Science.') and by the Netherlands Organization for Scientific Research (NWO) under a NWO-MaG Pionier grant.

Corresponding author: Cars Hommes, Center for Nonlinear Dynamics in Economics and Finance (CeNDEF), University of Amsterdam, Roetersstraat 11, NL-1018 WB Amsterdam, The Netherlands, Tel: ++31 20525 4246, Fax: ++31 20525 4349, e-mail: C.H.Hommes@uva.nl 


\section{Introduction}

Modern finance is based on the concept of rational expectations. As a consequence financial markets are considered to be efficient in the sense that past prices cannot help in predicting future prices. This view is known as the efficient market hypothesis (EMH). There has been a long debate about the EMH. In particular, there is empirical evidence that many 'stylized facts' observed in financial time series cannot solely be explained by fundamentals, but that markets have internal dynamics of their own.

One of the most important 'stylized facts' is volatility clustering. Whereas changes in asset prices themselves appear to be unpredictable, the magnitudes of those changes seem to be predictable in the sense that large changes tend to be followed by large changes - either positive or negative - and small changes tend to be followed by small changes. Asset price fluctuations are thus characterized by episodes of high volatility, with large price changes, irregularly interchanged by episodes of low volatility, with small price changes. Mandelbrot (1963) first discovered this phenomenon in commodity prices. Since the pioneering work of Engle (1982) and Bollerslev (1986) on autoregressive conditional heteroskedastic (ARCH) models and their generalization to GARCH models, volatility clustering has been shown to be present in a wide variety of financial assets including stocks, market indices and exchange rates. In empirical work, volatility clustering is usually modeled by a statistical model, such as the familiar (G)ARCH model or one of its extensions. Although these models are useful as a statistical description of the data, they do not offer a structural explanation of why volatility clustering is present in so many financial time series. Rather the statistical models postulate that volatility clustering has an exogenous source and is, for example, caused by the clustered arrival of random 'news' about economic fundamentals.

A recent branch of literature, including for example Arthur et al. (1997), Brock and Hommes (1997, 1998), Brock et al. (2005), Farmer and Joshi (2002), Gaunersdorfer and Hommes (2007), Kirman (1991), LeBaron (2001), LeBaron et al. (1999), Lux (1995, 1997) and Lux and Marchesi $(1999,2000)$, has offered a structural explanation of the phenomenon of volatility clustering by multi-agent systems, where financial markets are viewed as complex evolutionary systems between competing boundedly rational trading strategies; see Hommes (2006) and LeBaron (2006) for recent surveys. In these multi-agent systems two important classes of traders can be distinguished, fundamentalists and technical analysts, having different trading strategies and expectations about future prices of a risky asset. The fundamentalists believe that prices will move towards their fundamental rational expectations (RE) value, as given by the expected discounted sum of future dividends. In contrast, the technical analysts observe past prices and try to extrapolate historical patterns. The multi-agent systems are characterized by an irregular switching between phases of low volatility, where fundamentalists dominate the market and prices move close to the RE fundamental price, and phases of high volatility, where the market is dominated by technical trading with prices deviating from the fundamental price. Volatility clustering arises as an endogenous phenomenon, caused or amplified by the trading process itself through heterogeneity, adaptive learning, and the evolutionary interaction between fundamentalists and technical analysts. 
Most work on heterogeneous agent modeling is computationally oriented however, and few analytical results are available. In this paper we show by means of a simple, analytically tractable behavioral multi-agent model with evolutionary learning that two generic phenomena cause endogenous clustered volatility: coexistence of two attractors (a steady state and a limit cycle) and so-called intermittency on a strange attractor. We conjecture that these phenomena also play an important role in generating clustered volatility in the artificial stock markets in the computationally oriented literature mentioned above. Our model is an extension of the adaptive belief systems $(A B S)$ introduced by Brock and Hommes $(1997,1998)$, henceforth BH. An ABS is a present discounted value asset pricing model with heterogeneous beliefs. There are two trader types: fundamentalists, who believe that prices will move in the direction of the 'fundamental value', and trend followers or chartists, who extrapolate the latest observed price change. The fractions of the two different trader types change over time according to evolutionary fitness, as measured by utility from realized profits or, equivalently (as we will show), forecasting accuracy in the recent past. Chartists, however, also condition their forecasting rule upon price deviations from the RE fundamental price; that is, they will abandon their charts and switch to a fundamentalist market view when prices move away too far from the RE fundamental price.

The first phenomenon naturally suited to describe volatility clustering is coexistence of attractors. In particular, our evolutionary model exhibits coexistence of a stable (fundamental) steady state and a stable limit cycle. When buffeted with dynamic noise, irregular switching occurs between close to RE fundamental steady state fluctuations, where the market is dominated by fundamentalists, and large amplitude price fluctuations, where the market is dominated by chartists. It is important to note that coexistence of attractors is a generic and structurally stable phenomenon.

An important question is how a stable limit cycle around a locally stable steady state can arise. We will show that for our evolutionary learning model there is a simple mathematical answer: coexistence of a stable steady state and a stable limit cycle arises due to a so-called Chenciner or degenerate Hopf bifurcation. To an economist not familiar with bifurcation theory this may sound rather exotic, but a Chenciner bifurcation is a codimension two bifurcation, implying that it is not due to a special specification of our model but that it is a generic feature for nonlinear systems with two or more parameters; see Kuznetsov (1998) for a detailed and advanced mathematical treatment of bifurcation theory including all possible codimension one and codimension two bifurcations. Close to a Chenciner bifurcation point, there exists an open region in the parameter space for which a stable steady state and a stable limit cycle coexist. Occurrence of a local Chenciner bifurcation of the steady state is therefore a sufficient condition for the occurrence of certain global dynamic phenomena, in particular for the coexistence of a stable steady state and a stable limit cycle. The Chenciner local bifurcation point acts as an 'organizing center' of the dynamical behavior, implying global dynamic phenomena such as the creation or disappearance of stable limit cycles as a single model parameter is varied; see, for example, Saleh (2006, pp. 22-23) for a more detailed mathematical discussion. We will show that our heterogeneous agent model with evolutionary learning has a Chenciner bifurcation point in a two dimensional subspace of the parameter space. Application and detection of codimension two bifurcations in economic modeling are relatively new. One of the first applications of a codimension two bi- 
furcation in economics is Benhabib et al. (2001), who show that a continuous time model with active monetary policy rules exhibits a Bogdanov-Takens bifurcation. ${ }^{1}$ To our best knowledge, the present paper contains the first economic application of the Chenciner bifurcation.

There is a strikingly simple economic intuition why one should in fact expect coexistence of a stable RE fundamental steady state and a stable limit cycle in our simple evolutionary model, when chartists extrapolate trends only weakly and fundamentalists are only weakly stabilizing. When trend extrapolation is weak, the RE fundamental steady state will be locally stable because trend followers do not extrapolate small deviations from the RE steady state strongly enough for prices to diverge, and the price will therefore return to the RE steady state. However, even when trend extrapolation is weak, an upward price trend far away from the RE steady state will be reenforced, causing prices to deviate even further from the fundamental. Such a diverging upward price trend cannot continue forever, however, since trend followers condition their rule upon deviations from the RE fundamental price. When prices move too far away from the fundamental, as measured, for example, by a price-earnings ratio, technical analysts abandon their charts and switch to fundamentalists beliefs. The conditioning of technical trading upon market fundamentals thus ensures that the upward price trend will stop and eventually will reverse into a downward trend. If the fundamentalists are only weakly stabilizing, prices move only slowly downwards into the direction of the fundamental price. The fraction of trend followers will increase again, reenforcing the downward price trend. The downward trend will continue and prices decrease below the fundamental RE steady state price. Since trend followers condition their rule upon deviations from the RE fundamental price, at some point the downward price trend reverses into an upward trend. The fraction of trend followers increases again, reenforcing the upward price trend, and prices will overshoot the fundamental RE steady state. A full cycle around the locally stable RE fundamental steady state is then complete. This intuition suggests that the interaction and evolutionary switching between weak trend extrapolation and weakly stabilizing fundamental analysis may lead to coexistence of a locally stable RE fundamental steady state and a locally stable limit cycle far from that steady state. The paper will make this simple economic intuition rigorous for a simple, stylized behavioral and analytically tractable heterogeneous agent model.

A second endogenous phenomenon suited to describe volatility clustering is intermittency. The phenomenon of intermittency, as introduced by Pomeau and Manneville (1980), occurs when asset price fluctuations are moving on a strange, chaotic attractor characterized by phases of almost periodic fluctuations irregularly interrupted by sudden bursts of erratic fluctuations. In the evolutionary learning model studied here, intermittency is characterized by close to the (locally unstable) RE fundamental steady state fluctuations, suddenly interrupted by price deviations from the fundamental triggered by technical trading. Recent mathematical results on homoclinic bifurcations have shown that for nonlinear systems strange attractors are the rule rather than the exception. Stated more precisely, strange attractors are persistent in the sense

\footnotetext{
${ }^{1}$ A Bogdanov-Takens bifurcation for a continuous time system occurs when the Jacobian matrix at the steady state has a double eigenvalue 0 and certain higher order genericity conditions are satisfied. For a discrete time system a Bogdanov-Takens bifurcation is characterized by a double eigenvalue +1 . Close to such a BogdanovTakens bifurcation point, the discrete system exhibits chaos and strange attractors.
} 
that they typically occur for a positive Lebesgue measure set (i.e. a set of positive probability) of parameter values, see, for example, Palis and Takens (1993) for a mathematical treatment. Economic applications of strange attractor theory are fairly recent and include, for example, the overlapping generations economy in de Vilder (1996) and the 'hog cycle' or cobweb model with evolutionary learning in Brock and Hommes (1997).

A similar economic intuition as the one described above explains why one should expect intermittency on a strange attractor to occur in our evolutionary model when chartists are strong trend extrapolators and fundamentalists are strongly stabilizing. Strong trend extrapolators destabilize the fundamental RE steady state because trend followers strongly extrapolate small deviations from the fundamental steady state, leading to diverging prices and, say, an upward price trend. When prices diverge and move away far above the RE fundamental value, technical traders conditioning their charts upon market fundamentals will abandon their rule and the upward price trend will stop and reverse into a downward price trend. When fundamentalists are strongly stabilizing, prices will then quickly move into a small neighborhood of the RE fundamental steady state. Since the RE steady state is locally unstable in the presence of strong extrapolators, the story then repeats. This mechanism suggests that the interaction and evolutionary switching between strongly extrapolating technical trading and strongly stabilizing fundamental strategies causes the RE fundamental steady state to have a saddle-point structure, with a locally destabilizing force due to strong trend extrapolation and a globally stabilizing force due to fundamentalists. In fact, the evolutionary learning system with strong trend extrapolation and strongly stabilizing fundamentalists is close to having a homoclinic orbit and its associated complicated dynamical behavior.

An important critique from 'rational expectations finance' upon heterogeneous agent models using simple habitual rule of thumb forecasting rules is that 'irrational' traders will not survive in the market. For example, Friedman (1953) argues that irrational speculative traders would be driven out of the market by rational traders, who would trade against them by taking infinitely long opposite positions, thus driving prices back to fundamentals. In an efficient market, 'irrational' speculators would simply lose money and disappear from the market. However, for example, De Long et al. (1990) have shown that a constant fraction of noise traders may on average earn higher expected returns than rational or smart money traders and may survive in the market with positive probability. ${ }^{2} \mathrm{BH}(1997,1998)$ have also discussed this point extensively and stress the fact that in an evolutionary framework technical analysts are not 'irrational', but they are in fact boundedly rational, since in periods when prices deviate from the RE fundamental price, chartists make better forecasts and earn higher profits than fundamentalists. Speculative deviations from the fundamental price may in fact be triggered by short run profit opportunities for chartists. On average, technical analysts and fundamentalists may earn approximately equal profits, so that in general fundamentalists cannot drive chartists out of the market. See also the survey in Hommes (2006) for an extensive discussion of these points. Our evolutionary approach is also related to reinforcement learning in evolutionary game theory as, for example, in Börgers and Sarin (1997); Samuelson (1997) contains a nice survey of related

\footnotetext{
${ }^{2}$ An early example of a heterogeneous agent model is Zeeman (1974); other more recent examples include Frankel and Froot (1988), Kirman (1991), Chiarella (1992) and Brock (1993).
} 
evolutionary models in game theory. For related work on adaptive learning and motivation of bounded rationality, see, for example, Grandmont (1998), Sargent (1993, 1999) and Evans and Honkapohja (2001). Bullard (1994) and Schönhofer (1999) show how adaptive learning based upon ordinary least squares can lead to local instability, cycles and even chaos. In related empirical work Brock et al. (1992) have shown that simple technical trading rules applied to the Dow Jones Index may yield positive returns, suggesting extra structure above and beyond the EMH fundamental.

The paper is organized as follows. Section 2 describes the asset pricing model with fundamentalists and chartists. Section 3 presents a local stability analysis of the fundamental steady state and bifurcations of codimension one. Section 4 discusses the complete 2-D (two dimensional) bifurcation diagram of the Chenciner bifurcation, whereas section 5 presents the bifurcation diagram in our adaptive learning model. Section 6 investigates chaotic dynamics in our model, and finally, section 7 concludes.

\section{The model}

The model introduced here deviates from the asset pricing model with heterogeneous beliefs in BH (1998) in two ways. Firstly, we use a different evolutionary fitness measure, namely utility from realized profits or equivalently risk adjusted realized profits (instead of non-risk adjusted realized profits). Secondly, technical traders condition their charts upon price deviations from the RE fundamental benchmark price; that is, the fraction of technical traders will decrease when prices move far away from the fundamental price. Both deviations will be discussed in more detail below.

Agents trade in a market with one risky and one risk-free asset. The risk-free asset is completely elastically supplied at a gross return $R>1 . p_{t}$ denotes the price (ex-dividend) of the risky asset and $\left\{y_{t}\right\}$ the (stochastic) dividend process. The dynamics of wealth of investor type $h$ is described by

$$
\tilde{W}_{h, t+1}=R W_{h t}+\tilde{R}_{t+1} z_{h t},
$$

where $z_{h t}$ is the number of shares of the risky asset purchased at time $t$ and $\tilde{R}_{t+1}=\tilde{p}_{t+1}+$ $\tilde{y}_{t+1}-R p_{t}$ is the excess return per share. Variables carrying tildes denote random variables. Let $E_{t}$ and $V_{t}$ denote conditional expectation and conditional variance based on a publically available information set $\mathcal{F}_{t}$, such as past prices and dividends, and let $E_{h t}$ and $V_{h t}$ denote the 'beliefs' or forecasts of investor type $h$ about conditional expectation and variance.

\section{Equilibrium}

Assuming that investors are myopic mean-variance maximizers, the demand for shares $z_{h t}$ by type $h$ solves

$$
\max \left\{E_{h t} \tilde{W}_{h, t+1}-\frac{a}{2} V_{h t} \tilde{W}_{t+1}\right\}, \quad \text { i.e. } \quad z_{h t}=\frac{E_{h t} \tilde{R}_{t+1}}{a V_{h t} \tilde{R}_{t+1}}
$$


Here the nonnegative parameter $a$ characterizes risk aversion. Let $z_{s t}$ and $n_{h t}$ denote the supply of shares per investor and the fraction of investors of type $h$ at time $t$, respectively. Equilibrium of supply and demand implies

$$
\sum_{h} n_{h t} z_{h t}=z_{s t}
$$

Assuming constant supply of outside shares over time we may stick to the (equivalent) special case $z_{s t} \equiv 0 .{ }^{3}$ Further, we assume that dividends are independently and identically distributed (iid), in particular, $E_{t} \tilde{y}_{t+1} \equiv y^{*}$.

\section{Beliefs}

In the case where there is only one type of trader the equilibrium equation (2) reduces to

$$
R p_{t}=E_{t} \tilde{p}_{t+1}+y^{*} \text {. }
$$

In the standard case $R>1$ there is only one solution $p_{t}^{*} \equiv p^{*}=y^{*} /(R-1)$ that satisfies the "no bubbles' condition $\lim _{t \rightarrow \infty} E \tilde{p}_{t}^{*} / R^{t}=0$. This price, given as the discounted sum of expected future dividends, would prevail in a perfectly rational world and will be called the fundamental price.

We make some simplifying assumptions concerning the beliefs:

A1 The beliefs about future prices and dividends are assumed to be of the form

$$
E_{h t}\left(\tilde{p}_{t+1}+\tilde{y}_{t+1}\right)=E_{t}\left(\tilde{p}_{t+1}^{*}\right)+y^{*}+f_{h}\left(p_{t-1}, \ldots, p_{t-L}\right)=R p_{t}^{*}+f_{h}\left(p_{t-1}, \ldots, p_{t-L}\right),
$$

where $f_{h}$ is some deterministic function of past prices describing the beliefs of trader type $h$ about price deviations from the fundamental value. Further, this assumption implies that investors have homogeneous beliefs about future dividends $E_{h t}\left(\tilde{y}_{t+1}\right)=E_{t}\left(\tilde{y}_{t+1}\right)=y^{*}$. Hence all traders are able to derive the fundamental price $p_{t}^{*}$.

A2 The beliefs about conditional variances of the excess returns are assumed to be of the form

$$
V_{h t} \tilde{R}_{t+1}=V_{t} \tilde{R}_{t+1} \equiv \sigma^{2}, \quad \forall h, t .
$$

That is, beliefs about conditional variances are the same for all types and constant over time. ${ }^{4}$

We consider a model with two simple belief types,

$$
\begin{array}{ll}
E_{1, t+1} \equiv p_{1, t+1}^{e}=p^{*}+v\left(p_{t-1}-p^{*}\right), & 0 \leq v \leq 1 \\
E_{2, t+1} \equiv p_{2, t+1}^{e}=p_{t-1}+g\left(p_{t-1}-p_{t-2}\right), & g \in \mathbb{R} .
\end{array}
$$

\footnotetext{
${ }^{3}$ In the case $z_{s t}=$ constant, one can introduce a risk adjusted dividend $y_{t+1}^{\#}=y_{t+1}-a \sigma^{2} z^{s}$ and proceed in the same way; see Brock (1997).

${ }^{4}$ Gaunersdorfer (2000) studies the case of time varying (homogeneous) beliefs about conditional variances. She obtains similar bifurcation routes to complicated asset price fluctuations as in the case with constant beliefs. We therefore restrict ourselves here to this more simple case. Chiarella and He (2002) introduce heterogeneity in beliefs about variances.
} 
Traders of type 1 are 'fundamentalists', believing that tomorrow's price will move in the direction of the fundamental price $p^{*}$ by a factor $v$. When $v$ is close to 0 (1) fundamentalists believe prices to move quickly (slowly) towards its fundamental value $p^{*}$. Traders of type 2 are technical traders or chartists, deriving their beliefs from price histories. Our specification (4) is only a simple example of a technical trading rule using only the latest observed price and the latest observed price change. If $g>0$ these traders are trend followers, extrapolating the latest observed price change; if $g<0$ they are contrarians expecting a reversal of the latest price change. Given our assumptions, the equilibrium dynamics (2) reads as

$$
R p_{t}=\sum_{h=1}^{2} n_{h t} p_{h, t+1}^{e}+y^{*} .
$$

\section{Fractions}

Fractions $n_{h t}$ are updated according to past performance, conditioned upon the deviation of actual prices from the fundamental value. The evolutionary competition part of the updating rules closely follows BH (1997, 1998). The additional conditioning upon deviations from the fundamental is, for example, similar to and motivated by the Santa Fe computational artificial stock market in Arthur et al. (1997) and LeBaron et al. (1999).

In a first, evolutionary step, fractions are determined as discrete choice probabilities

$$
\hat{n}_{h t}=\exp \left[\beta U_{h, t-1}\right] / Z_{t}, \quad Z_{t}=\sum_{h} \exp \left[\beta U_{h, t-1}\right],
$$

where $U_{h t}$ is some 'fitness function' or 'performance measure'. Note that the fractions are independent of the fitness level; they do not change if the same term is added to the exponents. The parameter $\beta$ is called the intensity of choice. It measures how sensitive traders are to differences in performance of trading strategies. For $\beta=0$ fractions are fixed over time and are (in the case of only two different types) equal to $1 / 2$. In the limit of $\beta \rightarrow \infty$ all traders choose immediately the predictor with the best performance in the recent past. Thus, for finite, positive $\beta$, agents are boundedly rational in the sense that fractions of the predictors are ranked according to their fitness.

BH (1998) take (accumulated) realized net profits as the evolutionary fitness measure. Realized profit in period $t$, given by $\pi_{h t}=R_{t} z_{h, t-1}$, does not take into account, however, the risk taken to achieve this profit. Here we take accumulated risk adjusted realized profits as the fitness measure. Risk adjusted realized profit in period $t$ is given by

$$
\pi_{h t}=R_{t} z_{h, t-1}-\frac{a}{2} \sigma^{2} z_{h, t-1}^{2},
$$

where $z_{h, t-1}$ is the demand for the risky asset by trader type $h$ as before. Notice that maximizing expected wealth in (1) is equivalent to maximizing expected utility from profits in (7). Risk adjusted realized profits as the fitness measure is thus consistent with the investors' demand 
function derived from myopic mean-variance maximization of expected wealth. A straightforward computation shows that the risk adjusted profits fitness measure is equivalent to a constant times minus squared prediction errors, so that the evolutionary fitness simplifies to

$$
U_{h t}=-\frac{1}{2 a \sigma^{2}}\left(p_{t}-p_{h t}^{e}\right)^{2}+\eta U_{h, t-1},
$$

where the parameter $0 \leq \eta \leq 1$ represents 'memory strength' of the fitness measure. ${ }^{5}$

In the second step of the updating of fractions conditioning on deviations from the fundamental by the technical traders is modeled as

$$
\begin{aligned}
n_{2 t} & =\hat{n}_{2 t} \exp \left[-\left(p_{t-1}-p^{*}\right)^{2} / \alpha\right], \quad \alpha>0 \\
n_{1 t} & =1-n_{2 t} .
\end{aligned}
$$

According to (9) the fraction of technical traders decreases more as prices deviate further from their fundamental value $p^{*}$. This is motivated by the fact that technical traders are conditioning their charts upon price deviations from the fundamental. One may interpret the term $-\left(p_{t-1}-p^{*}\right)^{2} / \alpha$ as a penalty term in the fitness measure of technical traders. This penalty term ensures that speculative bubbles cannot last forever and explode to infinity, but that at some point when prices have moved far away from the fundamental value the fraction of fundamentalists will increase and stabilize prices. The penalty term ensures that price deviations from the fundamental remain bounded. ${ }^{6}$

Notice that fractions in period $t$ depend on observed prices up to the end of period $t-1$ (beginning of period $t), p_{t-1}, p_{t-2}, \ldots$

\footnotetext{
${ }^{5}$ See Gaunersdorfer (2001) and Hommes (2001) for a detailed computation showing this equivalence. Numerical simulations in Gaunersdorfer and Hommes (2007) suggest that the dynamics of the model with realized profits as the fitness measure is very similar to the analysis presented below.

${ }^{6}$ Hommes (2001) gives an interpretation of this 'penalty term' as a transversality condition in a heterogeneous world, where temporary speculative bubbles are allowed but price deviations from the fundamental remain bounded.
} 


\section{Dynamical system}

Setting $\hat{U}_{h t}=U_{h, t-1}$, we obtain the following dynamical system:

$$
\begin{aligned}
p_{t} & =\frac{1}{R}\left(p_{1, t+1}^{e}+n_{2 t}\left(p_{2, t+1}^{e}-p_{1, t+1}^{e}\right)+y^{*}\right) \\
\hat{U}_{h t} & =-\frac{1}{2 a \sigma^{2}}\left(p_{t-1}-p_{h, t-1}^{e}\right)^{2}+\eta \hat{U}_{h, t-1}, \quad h=1,2 .
\end{aligned}
$$

Introducing new variables $p_{i}(t-1)=p_{t-i}, u_{h}(t-1)=\hat{U}_{h, t-1},(10)-(11)$ is written as a 6-D system in $\mathbf{p}:=\left(p_{1}, p_{2}, p_{3}, p_{4}, u_{1}, u_{2}\right)$. In the following we denote this system by $\Phi$, where

$$
\mathbf{p}(t)=\Phi(\mathbf{p}(t-1))
$$

Also, when working in a neighborhood of $p^{*}$, it will be convenient if local coordinates $\mathbf{x}:=$ $\left(x_{1}, \cdots, x_{4}, u_{1}, u_{2}\right)$ are introduced by

$$
x_{i}(t)=p_{i}(t)-p^{*}
$$

where $x_{i}$ denotes the deviation from the fundamental price $p^{*}$. The system then takes the form

$$
\begin{aligned}
\Phi(\mathbf{x})= & {\left[\frac{1}{R}\left(\left(1-n_{2}\right) v x_{1}+n_{2}\left(x_{1}+g\left(x_{1}-x_{2}\right)\right)\right), x_{1}, x_{2}, x_{3}\right.} \\
& \left.\quad-\frac{1}{2 a \sigma^{2}}\left(x_{1}-v x_{3}\right)^{2}+\eta u_{1},-\frac{1}{2 a \sigma^{2}}\left(x_{1}-x_{3}-g\left(x_{3}-x_{4}\right)\right)^{2}+\eta u_{2}\right]
\end{aligned}
$$

where $n_{2}$ is given by

$$
n_{2}=\mathrm{e}^{-x_{1}^{2} / \alpha} \frac{\mathrm{e}^{\beta u_{2}}}{\mathrm{e}^{\beta u_{1}}+\mathrm{e}^{\beta u_{2}}} .
$$

\section{Stability analysis of the fundamental steady state}

This section gives a local analysis of the dynamics around the fundamental steady state. In the first part it is shown that $\mathbf{x}^{*}=0$ is the only steady state of (12). In the following, this will be called the fundamental steady state or the fundamental for short. The remainder of this section analyzes the stability of this fundamental steady state. It is stable if the trend parameter $g$ is close to 0 , and it loses its stability in two different ways: period doubling bifurcations occur for certain negative values of $g$, while Hopf bifurcations occur for some positive $g$. 


\subsection{Uniqueness and stability of the steady state}

The first lemma shows that the fundamental steady state with $p=p^{*}$, or equivalently $x^{*}=0$, is the unique steady state.

\section{Lemma 1}

Let $\Phi(\mathbf{x})$ be given by (12). Let moreover $R>1,0 \leq v \leq 1$ and $0 \leq \eta<1$. Then $\mathrm{x}^{*}=0$ is the unique steady state of $\Phi$.

\section{Proof}

Let $\mathrm{x}^{*}$ be any steady state of $\Phi$; that is, let $\mathrm{x}^{*}$ satisfy

$$
\mathbf{x}^{*}=\Phi\left(\mathbf{x}^{*}\right) \text {. }
$$

Notice first that $\mathbf{x}^{*}=\mathbf{0}$ is indeed a steady state. From the second, third and fourth component of equation (13), it follows that $x_{1}=x_{2}=x_{3}=x_{4}$. Setting $x_{1}=x$, the first component then reads as

$$
R x=\left(1-n_{2}\right) v x+n_{2} x .
$$

Assuming that $x \neq 0$, we may divide both sides of this equation by $x$, but then we have

$$
R=\left(1-n_{2}\right) v+n_{2} \leq 1<R,
$$

which is a contradiction, hence $x=0$. Now the last two components of equation (13) yield

$$
u_{1}=\eta u_{1}, \quad u_{2}=\eta u_{2}
$$

Since $\eta \neq 1,{ }^{7}$ the lemma follows.

\section{Stability}

In order to determine the stability of the fixed point, the characteristic polynomial of the Jacobian $D \Phi(\mathbf{0})$ at the steady state is computed. It is given by

$$
p(\lambda)=\lambda^{2}(\eta-\lambda)^{2}\left(\lambda^{2}-\frac{1+g+v}{2 R} \lambda+\frac{g}{2 R}\right) .
$$

Thus, the eigenvalues of the Jacobian are $0, \eta$ (both of multiplicity 2) and the roots $\lambda_{1}, \lambda_{2}$ of the quadratic polynomial in the last bracket. Note that these roots satisfy the relations

$$
\lambda_{1}+\lambda_{2}=\frac{1+g+v}{2 R} \quad \text { and } \quad \lambda_{1} \lambda_{2}=\frac{g}{2 R} .
$$

Also note that the eigenvalues 0 and $\eta$ always lie inside the unit circle. Thus, the stability of the steady state is determined by the absolute values of $\lambda_{1}$ and $\lambda_{2}$.

\footnotetext{
${ }^{7}$ For $\eta=1$ the dynamical system has a double eigenvalue 1 (see equation (14)) and hence is non-generic in a two parameter system. Though this is an interesting case, it is (because of additional mathematical difficulties) beyond the scope of this paper to analyze it.
} 


\subsection{Codimension one bifurcations}

As parameters are varied, bifurcations, that is, qualitative changes of the dynamical behavior, will arise. In particular, bifurcations changing the (local) stability of the fundamental steady state may occur. At such a bifurcation value, the steady state must be non-hyperbolic having (at least) one eigenvalue of $D \Phi(\mathbf{0})$ with absolute value one; that is, one of the eigenvalues is equal to $1,-1$, or there is a pair of complex eigenvalues on the unit circle. We first discuss the codimension one bifurcations, which are those bifurcations that are expected to occur (generically) when only a single parameter is varied.

\section{Eigenvalue equal to 1}

Assume that one of the eigenvalues $\lambda_{j}$ is equal to 1 , say $\lambda_{2}=1$. Then it follows from (15) that

$$
\lambda_{1}=\frac{g}{2 R} \quad \text { and } \quad 1+\lambda_{1}=\frac{1+g+v}{2 R} .
$$

Eliminating $\lambda_{1}$ from these equations leads to the condition

$$
1+v=2 R .
$$

However, since $v \leq 1<R$, this condition can never be satisfied. Hence eigenvalues equal to 1 cannot occur.

\section{Eigenvalue equal to -1}

Under the assumption that $\lambda_{2}=-1$, equations (15) lead to the relations

$$
\lambda_{1}=-\frac{g}{2 R} \quad \text { and } \quad-1+\lambda_{1}=\frac{1+g+v}{2 R} .
$$

Eliminating $\lambda_{1}$ leads to

$$
2 g+v=-1-2 R .
$$

For parameters satisfying this equation, a period-doubling (also called flip) bifurcation of the steady state is found (if a certain non-degeneracy condition is satisfied).

\section{Two complex conjugate eigenvalues of modulus 1}

The roots $\lambda_{1}, \lambda_{2}$ of the characteristic equation are complex conjugate and of modulus one if $\lambda_{1} \lambda_{2}=1$ and $\left|\lambda_{1}+\lambda_{2}\right|<2$. Using (15), this leads to the conditions

$$
\frac{g}{2 R}=1 \quad \text { and } \quad\left|\frac{1+g+v}{2 R}\right|<2 .
$$

Substituting the first condition into the second yields

$$
\left|1+\frac{1+v}{2 R}\right|<2
$$

For $0 \leq v \leq 1$, this condition is always satisfied, since $R>1$. Hence, for parameters satisfying the equation

$$
g=2 R
$$


a Hopf (also called Neimark-Sacker) bifurcation occurs (again if certain non-degeneracy conditions are satisfied).

\section{Conclusion}

Introduce the functions $g_{P D}(\beta, v, R)$ and $g_{H}(\beta, v, R)$ by

$$
\begin{aligned}
g_{P D}(\beta, v, R) & =-\frac{1}{2}(v+2 R+1) \\
g_{H}(\beta, v, R) & =2 R .
\end{aligned}
$$

The following lemma summarizes the above discussion.

\section{Lemma 2}

The steady state $\mathrm{x}^{*}=\mathbf{0}$ is hyperbolic for $g \neq g_{P D}$ and $g \neq g_{H}\left(g_{P D}\right.$ and $g_{H}$ defined by (16) resp. (17)). It is asymptotically stable for $g_{P D}<g<g_{H}$, and unstable for $g>g_{H}$ and $g<g_{P D}$.

For $g=g_{P D}$ or $g=g_{H}$, the fundamental steady state fails to be hyperbolic. In the first case $D \Phi(0)$ has an eigenvalue -1 , in second case two complex conjugate eigenvalues of absolute value 1 occur.

If moreover in these latter cases certain non-degeneracy conditions are satisfied, then for $g=$ $g_{P D}$ the system undergoes a period doubling (flip) bifurcation, and for $g=g_{H} a$ Hopf (Neimark-Sacker) bifurcation.

Notice that the period doubling bifurcation value $g_{P D}<0$, so that a period doubling bifurcation only occurs in the presence of contrarians who expect a reversal of the latest price change. Since $v \geq 0$ and $R>1$ it follows that $g_{P D}<-1.5$ so that only strong contrarian behavior can destabilize the fundamental steady state. The Hopf bifurcation value $g_{H}>0$ and is thus caused by trend extrapolating behavior. Since $R>1$ it follows that $g_{H}>2$, implying that only strong trend extrapolators can destabilize the fundamental steady state. ${ }^{8}$ In the next section we show however that even for intermediate trend following parameters $1<g<2$, although the fundamental steady state is locally stable, our evolutionary system can have a coexisting stable limit cycle or even a coexisting strange attractor.

\section{A Chenciner bifurcation}

In the last two decades, economists have become familiar with period doubling and Hopf bifurcations. These are examples of codimension one bifurcations; that is, these bifurcations occur generically when a single system parameter is varied. Economic applications of bifurcations of codimension two or higher are rare however, probably because they are more difficult to handle.

\footnotetext{
${ }^{8}$ Gaunersdorfer (2001) introduces positive per period costs for information gathering by fundamentalists. When fundamentalists' beliefs are costly compared to simple technical trading rules, the period doubling and Hopf bifurcation values move closer to 0 , and a period doubling bifurcation may already occur for $g_{P D} \approx-1$ and a Hopf bifurcation already for $g_{H} \approx 1$.
} 
An exception is in Benhabib et al. (2001) who find a Bogdanov-Takens bifurcation point, that is a bifurcation with a double eigenvalue 0 , in a continuous time model with active monetary policy rules.

In this section we discuss an important codimension two bifurcation, the so-called Chenciner bifurcation (also called degenerate Hopf bifurcation), that occurs in our heterogeneous agent model with evolutionary learning. A codimension two bifurcation is a non-generic phenomenon when only one parameter is varied, but it is a generic phenomenon when two parameters are varied simultaneously. The importance of the Chenciner bifurcation for our evolutionary learning model is the fact that close to a Chenciner bifurcation point there is an open region in the parameter space where a stable steady state and a stable limit cycle coexist. We will identify such a region (which we call a 'volatility clustering region') in the $g$ - $v$-parameter space in our model (see section 5, figure 3).

This section presents a general discussion of the Chenciner bifurcation by means of the 2-D bifurcation diagram of its normal form (see figure 1). The normal form of a bifurcation may be thought of as the simplest model in which the bifurcation occurs. Section 5 then shows that the Chenciner bifurcation occurs in our evolutionary learning model and discusses the bifurcation diagram for our adaptive belief system.

As discussed in section 3, a steady state loses stability through a Hopf bifurcation when its Jacobian matrix has two eigenvalues on the unit circle with all other eigenvalues inside the unit circle. There are two types of Hopf bifurcation:

(i) a supercritical Hopf bifurcation where the stable steady state becomes unstable, and the unstable steady state is surrounded by an attracting invariant circle with periodic or quasiperiodic dynamics; and

(ii) a subcritical Hopf bifurcation where the stable steady state becomes unstable, and the stable steady state is surrounded by a repelling invariant circle with periodic or quasiperiodic dynamics.

The normal form of the Hopf bifurcation determines whether it is super- or subcritical. Such a normal form can be obtained by a (sequence of) suitable coordinate transformation(s) around the steady state, restricted to the center manifold, which is an invariant manifold through the steady state tangent to the eigenspace spanned by the eigenvectors associated to the complex eigenvalues $\lambda$ and $\bar{\lambda}$. The normal form of a Hopf bifurcation is a 2-D map describing the dynamics on the center manifold. Although such normal form computations are straightforward, in practical applications they can be quite complicated. For general mathematical references on bifurcations theory and details on how to compute center manifolds and normal forms see, for example, Guckenheimer and Holmes (1986) and Kuznetsov (1998).

The normal form of the Hopf bifurcation with complex eigenvalues $\lambda=(1+\mu) e^{i \omega}$, written in polar coordinates, has the following general form:

$$
\varphi(r, \vartheta)=\left(r+\mu r+\nu r^{3}, \vartheta+\omega+\gamma r^{2}\right)+\ldots
$$


Here the dots denote terms of higher order in $r$ and $\vartheta$. Polar coordinates $(r, \vartheta)$ are used to describe points on the 2-D real center manifold $W^{c}$. They are chosen such that $r=0$ corresponds to the steady state of the system. The Hopf bifurcation occurs at $\mu=0$, for which the complex eigenvalues lie on the unit circle and $\omega$ denotes the angle of the complex eigenvalues. For a generic Hopf bifurcation, the coefficients $\nu$ and $\gamma$ must satisfy the non-degeneracy condition $\nu \neq 0 \neq \gamma$. The non-degeneracy condition $\gamma \neq 0$ ensures that the rotational part of the normal form is not linear. For $\nu<0$ the Hopf bifurcation is supercritical, whereas for $\nu>0$ the Hopf bifurcation is subcritical. In applications, the coefficient $\nu$ depends upon system parameters, and its computation can be quite complicated.

For $\nu=0$ the Hopf bifurcation becomes degenerate, and higher order terms have to be taken into account in the analysis. This bifurcation is called a degenerate Hopf or Chenciner bifurcation, and has been analyzed originally by Chenciner (1985a, 1985b, 1988); see also Kuznetsov (1998) for a textbook treatment. The Chenciner bifurcation is a codimension two bifurcation, implying that it is a generic phenomenon in systems with two or more parameters.

The normal form of the Chenciner bifurcation with complex eigenvalues $\lambda=(1+\mu) e^{i \omega}$ and written in polar coordinates is given by

$$
\varphi(r, \vartheta)=\left(r+\mu r+\nu r^{3}+\gamma_{1} r^{5}+\ldots, \vartheta+\omega+\gamma_{2} r^{2}+\ldots\right) .
$$

Here the dots again denote terms of higher order in $r$ and $\vartheta$. The Chenciner bifurcation occurs at $(\mu, \nu)=(0,0)$ for which the complex eigenvalues lie on the unit circle and the third order term in the normal form vanishes. The non-degeneracy conditions for the Chenciner bifurcation are in these coordinates $\gamma_{1} \neq 0 \neq \gamma_{2}$. We discuss the case $\gamma_{1}<0$ (which occurs in our application in section 5) and, without loss of generality, we assume that $\gamma_{1}(0)=-1$. See Kuznetsov for more information. The normal form then simplifies to

$$
\varphi(r, \vartheta)=\left(r+\mu r+\nu r^{3}-r^{5}, \vartheta+\omega+\gamma_{2} r^{2}\right),
$$

where the higher order terms are set to zero.

We discuss the structure of the local bifurcation diagram of the Chenciner bifurcation, illustrated in figure 1, using the normal form (20). Note that any positive solution $r_{*}$ to the equation

$$
\mu+\nu r^{2}-r^{4}=0
$$

or, equivalently, to

$$
\left(r^{2}-\frac{\nu}{2}\right)^{2}=\frac{\nu}{4}^{2}+\mu
$$

corresponds to an invariant circle in phase space.

[Figure 1 about here.]

For $\mu>0$, equation (21) has exactly one positive solution. For $\mu=0$ equation (21) has a solution $r_{*}=0$. Thus, $\mu=0$ is a line of Hopf bifurcations, whose type is determined by the sign of $\nu$ : for $\nu<0$, the Hopf bifurcation is supercritical, for $\nu>0$ it is subcritical. 
The number of positive solutions for $\mu<0$ is determined by the sign of $\nu^{2} / 4+\mu$ : there are two if it is positive, none if it is negative. Finally, for parameters on the curve

$$
S N: \frac{\nu^{2}}{4}+\mu=0
$$

two positive roots of equation (21) coincide. The curve $S N$ in (22) thus corresponds to parameter values for which a saddle-node bifurcation of invariant circles occurs.

A sketch of the complete bifurcation diagram is given in figure 1. Consider a point in parameter space $\{(\mu, \nu)\}$, with $\mu<0$ and $\nu<0$. For these parameter values the steady state is locally stable. Now fix $\nu$ and increase $\mu$. When crossing the negative $\nu$-axis, for $\mu=0$, a supercritical Hopf bifurcation occurs; that is, a stable invariant circle is created and the steady state becomes unstable. Thus, in the region $\{\mu>0\}$ a stable limit cycle around an unstable steady state exists. Now fix a parameter value $\nu>0$ and decrease $\mu$ from some positive value. When crossing the positive $\nu$-axis at $\mu=0$, a subcritical Hopf bifurcation occurs in which the steady state becomes stable, an unstable invariant circle emerges out of the steady state, and the stable invariant circle still exists. Decreasing $\mu$ further, the unstable and stable circles approach each other and dissappear in a saddle-node bifurcation of invariant circles when $\mu$ crosses the curve $S N$. Thus, in the region between the positive $\nu$-axes and the curve $S N$ the system has two attractors, a stable steady state and an attracting (large) invariant circle, separated by an unstable invariant circle that forms the boundary between these two attractors. We will call this region a 'volatility clustering region', since adding some noise to the system, the dynamics is characterized by an irregular switching between phases of small amplitude fluctuations close to the steady state with small changes and phases of large amplitude fluctuations with large changes along the limit cycle. The boundary of the volatility clustering region is formed by the curve of supercritical Hopf bifurcations of the steady state (the positive $\nu$-axis) and the curve $S N$ of saddle-node bifurcations of an invariant circle, and these boundary curves all end at the Chenciner bifurcation point in the origin.

Finally, we note that the dynamics on the invariant circles may undergo bifurcations as well. For these dynamics there are two possibilities. The first possibility is that the dynamics on these circles consist of a sequence of attracting and repelling hyperbolic periodic points. This type of dynamics is called resonating, phase locked or Morse-Smale; for an example see figure 4 (middle plot). There is usually an open set of parameters for which the invariant circle has phase locked dynamics. The boundaries of this set are formed by saddle-node bifurcation curves of the attracting and repelling points on the invariant circle. ${ }^{9}$ While the total set of parameters with phase locked dynamics is open and dense, its complement has positive measure. Parameters in the complement correspond to the case that the dynamics on the invariant circle are quasiperiodic; an example is shown in the left plot of figure 4. There is a large literature on quasiperiodic dynamics, to which the interested reader is referred (see Moser 1973, Herman 1979, Arnol'd 1983, Broer et al. 1990, and references there).

\footnotetext{
${ }^{9}$ Pintus et al. (2000) present an infinite horizon intertemporal equilibrium model exhibiting these types of local bifurcations after a Hopf bifurcation of the steady state, finally leading to strange attractors.
} 
The local, codimension two Chenciner bifurcation point acts as an 'organizing center' for the dynamical behaviour for nearby parameter values. In particular, existence of a local, codimension two Chenciner bifurcation of the steady state, with the sign restriction $\gamma_{1}<0$ in the normal form (19), implies a global, codimension one saddle-node bifurcation of invariant circles and the existence of a 'volatility clustering' region, where a stable steady state and a stable limit cycle coexist in the parameter space. Close to the Chenciner bifurcation point the dynamics on the stable invariant circle is either periodic or quasi-periodic. When parameters move away from the Chenciner bifurcation point subsequent bifurcations may lead to more complicated, chaotic dynamics. As we will see in section 6 (see e.g. the right plot in figure 4), this may happen even when the steady state remains locally stable.

\section{The onset of instability}

This section investigates the onset of instability in our simple behavioral model with evolutionary learning. In particular, we investigate the following question: what is the set of parameter values for which prices in our heterogeneous agent model with evolutionary learning do not necessarily converge to the fundamental steady state? It turns out that, even when the fundamental steady state is locally stable, prices need not converge to their fundamental value, but may settle down to a stable limit cycle. In particular, a Chenciner bifurcation with a bifurcation diagram similar to figure 1 plays an important role in our model with evolutionary learning.

Our adaptive learning model is determined by the mapping $\Phi$ in (12). To simplify the computations we restrict our analysis to the case of no memory in the performance measure $(\eta=0)$, so that the dimension of the state space of the system reduces from six to four. The parameter space $P$ is equal to ${ }^{10}$

$$
P=\{(\beta, v, g, R): \beta>0,0 \leq v \leq 1, R>1\} .
$$

Recall from lemma 2 in section 3 that a Hopf bifurcation occurs for $g=2 R$. The Hopf bifurcation manifold for our system (12) is therefore given by

$$
H=\{(\beta, v, g, R) \in P: g=2 R\} .
$$

Within the codimension one Hopf bifurcation manifold $H$, one can find Chenciner bifurcation points by changing a second parameter, different from $g$, until the Hopf bifurcation becomes degenerate, that is, until the coefficient $\nu$ in the normal form (18) of the Hopf bifurcation becomes 0 . For our evolutionary adaptive learning model, the locus of Chenciner bifurcation points in the 2-D $\beta$ - $v$-parameter plane, within the Hopf bifurcation manifold $H=\{g=2 R\}$,

\footnotetext{
${ }^{10}$ Changing the values of parameters $a$ and $\sigma$ is equivalent to choosing a different value for $\beta$, see equations (6) and (8). Further, by changing to new coordinates $\mathbf{x}=\sqrt{\alpha} \tilde{\mathbf{x}}$, the iteration equation (12) changes to $\sqrt{\alpha} \tilde{\mathbf{x}}_{t+1}=$ $\Phi\left(\sqrt{\alpha} \tilde{\mathbf{x}}_{t}\right)$ and it follows, by some algebra, that $\tilde{\mathbf{x}}_{t+1}=\Phi\left(\tilde{\mathbf{x}}_{t}\right)$, where the parameter $\beta$ is replaced by $\tilde{\beta}=\alpha \beta$. Thus, attention may be reduced to the case $\alpha=1$. Hence we can restrict the parameter space of our model to $P$.
} 
is plotted in figure $2 .{ }^{11}$ Figure 2 shows that for $0.3 \leq \beta<3.3$ two Chenciner bifurcation points exist, with the largest $v$-value approaching 1 as $\beta$ approaches 3.3. For larger $\beta$-values, $\beta>3.3$, a Chenciner bifurcation point occurs for $v$-values around 0.35 .

[Figure 2 about here.]

[Figure 3 about here.]

Figure 3 (left panel) shows the bifurcation diagram around the Chenciner bifurcation point in the 2-D $g-v$-parameter plane of our adaptive learning system, for $R=1.01$ and $\beta=100$; the reader should compare this figure to the $\mu-\nu$-bifurcation diagram of the normal form of the Chenciner bifurcation in figure 1 . The Chenciner bifurcation point, lying on the Hopf bifurcation manifold $H$, is labelled $D H$, whereas the curve labelled $S N$ is the saddle-node bifurcation curve of the invariant circles. The volatility clustering region lies between the curve $S N$ and the Hopf bifurcation manifold $H$. In this region a second attractor (a stable limit cycle, or possibly a more complicated, chaotic attractor) coexists with the stable fundamental steady state. Below the Chenciner bifurcation point the Hopf bifurcation is supercritical; above the Chenciner bifurcation point the Hopf bifurcation is subcritical. Numerical simulations suggest that on the left hand side of or below the curve $S N$, the fundamental steady state is globally stable. When crossing the curve $S N$ from left to right, a pair of invariant circles, one stable and one unstable, are created. The unstable invariant circle separates the stable fundamental steady state from the stable invariant circle. The curve $S N$ thus marks the onset of instability. To the right of this curve, prices do not necessarily converge to their fundamental value but may converge to a stable limit cycle or to a more complicated, chaotic attractor. The enlargement in the right panel of figure 3 shows that, as the intensity of choice $\beta$ to switch strategies increases, the curve $S N$ moves to the left and approaches the vertical line $g=1$. This implies that although the fundamental steady state remains locally stable, for higher values of the intensity of choice $\beta$, (global) instability sets in already for a trend parameter $g$ close to 1.

The saddle-node bifurcation of the invariant circle is a 'global' phenomenon, in the sense that invariant circles are 'global' objects, and it typically occurs 'far away' from the steady state. Except in small neighborhoods of Chenciner bifurcation points, no analytic information can be obtained about the location of the manifold $S N$. The sketch of the location of $S N$ in figure 3 has been obtained as follows. For fixed values of $\beta$ and $v$, plots of the phase space have been inspected numerically for a range of $g$-values. The lowest value of $g$ (to a precision of 0.001 ) for which an attractor other than the fundamental steady state existed has been termed the (approximate) saddle-node bifurcation value $g_{*}(\beta, v)$ of the invariant circle. The enlargement in the right panel of figure 3 shows that the curve $S N$ of $g_{*}$ moves to the left and approaches $g=1$ as the intensity of choice $\beta$ increases (see also Gaunersdorfer et al. 2005 for a plot in the 3-D parameter space $\{(g, v, \beta)\})$.

\footnotetext{
${ }^{11}$ This is a nontrivial figure, based upon more than 10 pages of algebraic computations of the normal form of the Hopf and the Chenciner bifurcations for our 4-D adaptive learning system; see appendix A in an earlier working paper Gaunersdorfer et al. (2000).
} 
The main economic consequence from this analysis is that if traders' sensitivity to differences in fitness is high (i.e. the intensity of choice $\beta$ is high) then the interaction between weakly extrapolating trend followers (i.e. for trend parameters $g$ close to 1 ) and weakly stabilizing fundamentalists (i.e. with $0.6 \leq v \leq 0.9$, say) leads to coexistence of attractors and agents may coordinate on a stable limit cycle around the locally stable fundamental steady state. ${ }^{12}$ In the presence of noise, the evolutionary dynamics then switches irregularly between a stable phase with close to the fundamental steady state small amplitude price fluctuations and an unstable phase with large price fluctuations along a (noisy) limit cycle. Our simple evolutionary learning model thus exhibits volatility clustering.

\section{The amount of chaos}

In section 3 it has been shown that in the case of $g>0$, the fixed point $\mathbf{x}^{*}=0$ is stable for small $g$, and it loses its stability in a Hopf bifurcation for $g=2 R$. In section 5 we have seen that a stable limit cycle may already exist for $g<2 R$ (in fact when $g$ is close to 1 ) when the fundamental steady state is still locally stable. In this section we focus on more complicated, chaotic dynamical behavior in the model and investigate for which parameter constellations chaos arises. In particular, we find another mechanism for clustered volatility in our adaptive belief system, namely intermittent chaos.

[Figure 4 about here.]

[Figure 5 about here.]

Figure 4 shows typical examples of attractors in the $p_{t}-p_{t+1}$-plane, for three different $g$-values with all other parameters fixed. Since $g<2 R$ these attractors coexist with a locally stable fundamental steady state. For $g=1.6$ an attracting quasi-periodic circle occurs, whereas for $g=1.7$ a stable limit cycle of period 16 occurs. For $g=2$, after a complicated sequence of bifurcations, the invariant circle has turned into a strange attractor. The numerical simulations in figure 4 thus suggest that in our adaptive learning model, a strange attractor with chaotic dynamical behavior may coexist with a locally stable fundamental steady state. In figure 4 (right plot), the unstable invariant circle created at the Chenciner bifurcation can be seen as the inner boundary between the strange attractor and the locally stable fundamental steady state.

Figure 5 illustrates what happens after the supercritical Hopf bifurcation in the model. An important difference between the figures is that the parameter $v$, that is, the factor with which fundamentalists expect prices to move towards the fundamental value, has been decreased from $v=0.6$ in figure 4 to $v=0.3$ in figure 5. Numerical simulations suggest that for $g=2$ the

\footnotetext{
${ }^{12}$ Hommes et al. (2005) have recently carried out laboratory forecasting experiments using a similar asset pricing framework. In these experiments both possibilities, with human subjects either learning to coordinate on the fundamental price or learning to coordinate on an oscillatory pattern, have been observed.
} 
fundamental steady state is globally stable, whereas for $g=2.09$ an invariant attracting circle, quasi-periodic (or periodic with high period), has appeared. For $g=2.4$, the invariant circle has developed into a strange attractor. Notice that the strange attractor in figure 5 (right plot) seems to contain the (unstable) fundamental steady state, suggesting that price fluctuations get close to the fundamental steady state occasionally. The corresponding chaotic time series suggests some form of volatility clustering caused by intermittent chaos, characterized by phases of growing prices and phases of fluctuations close to the fundamental price.

There is a strikingly simple economic intuition why such intermittent chaos may in fact be expected when chartists are strong trend extrapolators (i.e. the trend parameter $g$ is large) and fundamentalists are strongly stabilizing (i.e. the parameter $v$ is close to zero). In the presence of strong trend extrapolators the fundamental steady state is locally unstable because trend followers strongly extrapolate small deviations from the fundamental steady state, leading to oscillatory, diverging prices. When prices diverge and move away far above or below the fundamental value, technical traders conditioning their charts upon market fundamentals will abandon their rule, the upward or downward price trend will stop, and most technical analysts will start following the fundamental rule. When fundamentalists are strongly stabilizing, prices will then quickly move into a small neighborhood of the fundamental steady state. Due to the strong trend extrapolators, the fundamental steady state is locally unstable, prices start oscillating again, and the story then repeats. This mechanism suggests that the evolutionary interaction causes the fundamental steady state to have a saddle-point structure with a locally destabilizing force due to strong trend extrapolation and a globally stabilizing force due to strong stabilization by fundamentalists.

This economic intuition suggests that the evolutionary learning system is in fact close to having a homoclinic orbit and its associated complicated dynamical behavior. The economic intuition also suggests reasons why the dynamics might be chaotic. A set of initial states of the system close to the fundamental will be stretched out during the phase when technical traders dominate and extrapolate a trend. At the point where the fundamentalists start to become the dominating fraction in the market, the set will be folded back onto itself. The action of the fundamentalists transports this folded set back close to the fundamental. It is precisely this stretching and folding that lies at the root of the occurrence of chaos in dynamical systems in general. Technical trading causes stretching, whereas the conditioning of technical trading rules upon fundamentals causes folding, and the interaction between these competing strategies create intermittent chaos in the adaptive belief system.

To get a global impression of the 'amount of chaos' to be expected in the system, Lyapunov exponents are computed for a range of parameter values.

\section{Definition}

If $\left\{\mathbf{x}_{t}\right\}_{t=0}^{\infty}$ is an orbit on an attractor, then the first (or largest) Lyapunov exponent $\lambda$ is defined as

$$
\lambda=\lim _{t \rightarrow \infty} \frac{1}{n} \sum_{k=0}^{n-1} \log \left\|D \Phi\left(\mathbf{x}_{t}\right)\right\| .
$$


The (largest) Lyapunov exponent measures the average rate of divergence (or convergence) of nearby trajectories. A system is commonly considered to be chaotic if it has an attractor such that orbits on the attractor have positive largest Lyapunov exponent.

For the present system, Lyapunov exponents have been computed for 10 different initial conditions, in order to account for the possibility of coexisting attractors. The largest value of the exponent obtained has been taken. These plots have been made both in the $g$ - $v$ - and the $\beta$ - $g$ diagrams (figures 6 and 7), given as a contour plot (right plots: region with positive Lyapunov exponents are indicated) and a 3-D-plot (left plots: $z$-coordinate indicates magnitude of the Lyapunov exponent). For $g>2 R$ chaos seems to be the rule rather than the exception, but even for the locally stable region of the fundamental steady state, $g<2 R$, for parameters in the "volatility clustering region' a coexisting chaotic attractor may exist (an example was shown already in the right plot of figure 4). In particular, chaos may arise for relatively small values of the trend parameter $g$ when the intensity of choice $\beta$ is large, that is, when traders are sensitive to small differences in fitness and quickly adapt their trading strategies.

[Figure 6 about here.]

[Figure 7 about here.] 


\section{Concluding Remarks}

In this paper we present a simple, nonlinear structural model for volatility clustering, based on the concept of evolutionary adaptive belief systems introduced by Brock and Hommes (1997). Volatility clustering arises endogenously due to the interaction between fundamentalists and technical analysts driven by adaptive learning. Two mechanisms are proposed as an explanation: intermittency and coexistence of attractors. Chaos arises from the combination of stretching due to strong trend extrapolation by chartists, folding due to conditioning of the chartists' forecasting rules upon market fundamentals, and contraction due to strong fundamentalists pushing prices quickly back close to the (unstable) fundamental steady state. Coexistence of attractors arises due to a codimension two Chenciner bifurcation. Close to the Chenciner bifurcation there is a 'volatility clustering region', that is, an open set in parameter space where a locally stable fundamental steady state and a stable invariant circle coexist. Both mechanisms proposed are generic phenomena and thus may serve as endogenous explanations of volatility clustering in more complicated computational multi-agent systems. The local, codimension two Chenciner bifurcation point acts as an 'organizing center' of the bifurcation structure and implies global, codimension one bifurcation phenomena such as a saddle-node bifurcation of invariant circles.

We have presented a strikingly simple economic intuition why in our heterogeneous agent adaptive learning model one should in fact expect both coexistence of a locally stable fundamental steady state and a stable limit cycle and intermittent chaos to occur, depending upon the strength of trend extrapolation (as measured by the trend parameter $g$ ) and the strength of fundamental stabilization (as measured by the factor $v$ with which fundamentalists expect prices to move towards the fundamental value).

In the presence of strongly extrapolating chartists small price deviations from the fundamental will be reenforced by trend extrapolation. The fundamental steady state will therefore be locally unstable and prices move away from their fundamental value. When the price deviation from the fundamental becomes too large however, chartists will abandon their rules since they condition their charts on market fundamentals. Most technical traders will thus start following a fundamental rule and, when fundamentalists are strongly stabilizing, prices will quickly move back close to the fundamental value, and the story repeats. Evolutionary interaction between strongly extrapolating chartists and strongly stabilizing fundamentalists leads to a strange attractor with intermittent chaos and irregular price fluctuations switching between phases of low and high volatility, as illustrated in figure 5.

When the chartists are only weak trend extrapolators, the fundamental steady state is locally stable. The trend extrapolators may be strong enough however to reenforce a price trend far away from the fundamental steady state. This upward trend, say, cannot continue forever, since chartists condition their rule upon market fundamentals and at some point will switch to become fundamentalists. Prices will then return in the direction of the fundamental value, and a downward trend will start. If fundamentalists are only weakly stabilizing, prices will only move slowly downwards and will not get close enough to the (locally stable) fundamental steady state. Trend followers will extrapolate the downward trend, until the point when prices move too far 
away from their fundamental value and the downward trend will be reversed into an upward trend, and the story repeats. This mechanism leads to a market in which a locally stable fundamental steady state coexists with a stable limit cycle. When the intensity of choice to switch strategies is high, coordination on a stable limit cycle around a locally stable fundamental steady state may arise even when trend followers are only weakly extrapolating (i.e. for $g$-values close to 1). When traders are highly sensitive to differences in fitness, the evolutionary interaction between weak trend extrapolators and weakly stabilizing fundamentalists may thus lead to a stable limit cycle (or a more complicated attractor) around a (locally) stable fundamental steady state. In the presence of noise, the market then switches irregularly between phases of low volatility and phases of high volatility, as illustrated in figure 8. A noise term (normal distribution, with standard deviation $\sigma=0.5$ ) has been added to the equilibrium pricing equation (10). Notice that this is equivalent to adding a small fraction of noise traders, who trade randomly, to the market clearing equation (2). Due to the presence of the noise traders, the market switches irregularly between a low volatility phase, dominated by fundamentalists with prices close to the fundamental value, and a high volatility phase, dominated by trend followers with prices exhibiting temporary bubbles. Temporary bubbles are triggered by noise traders and reenforced by trend followers.

[Figure 8 about here.]

The model studied here is admittedly simple and should only be viewed as a stylized, analytically tractable behavioral model. Volatility clustering arises, at least in a qualitative sense. We have proposed intermittency and the coexistence of attractors (e.g. arising from a Chenciner bifurcation) as an endogenous explanation of clustered volatility. Both intermittency and the coexistence of attractors are 'generic' phenomena, and similar phenomena are expected to occur in more complicated, nonlinear dynamic models.

A convenient feature of our simple adaptive belief system is that the model has been formulated around a benchmark fundamental. In this paper we have focused exclusively on the case of a constant fundamental, derived from an underlying iid dividend process. A natural extension is to investigate the evolutionary adaptive system in the case of a more realistic stochastic dividend process and its corresponding time varying stochastic fundamental processes, for example a geometric random walk, to see whether simple stochastic models can match the observed volatility clustering in real financial data more closely. 


\section{References}

Arnol'd, V.I., 1983. Geometric methods in the theory of ordinary differential equations. Springer: New York.

Arthur, W.B., Holland, J.H., LeBaron, B., Palmer, R., Taylor, P., 1997. Asset pricing under endogenous expectations in an artificial stock market. In: Arthur, W.B., Durlauf, S.N., Lane, D.A. (Eds.), The economy as an evolving complex system II. Addison-Wesley: Redwood City, 15-44.

Benhabib, J., Schmitt-Grohé, S., Uribe, M., 2001. The perils of Taylor rules. Journal of Economic Theory 96, 40-69.

Bollerslev, T., 1986. Generalized autoregressive conditional heteroscedasticity. Journal of Econometrics 31, 307-327.

Börgers, T., Sarin, R., 1997. Learning through reinforcement and replicator dynamics. Journal of Economic Theory $77,1-14$.

Brock, W.A., 1993. Pathways to randomness in the economy: emergent nonlinearity and chaos in economics and finance. Estudios Económicos 8, 3-55.

Brock, W.A., 1997. Asset price behavior in complex environments. In: Arthur, W.B., Durlauf, S.N., Lane, D.A. (Eds.), The Economy as an Evolving Complex System II. Addison-Wesley: Reading, MA, 385423.

Brock, W.A., Hommes, C.H., 1997. A rational route to randomness. Econometrica 65, 1059-1095.

Brock, W.A., Hommes, C.H., 1998. Heterogeneous beliefs and routes to chaos in a simple asset pricing model. Journal of Economic Dynamics and Control 22, 1235-74.

Brock, W.A., Hommes, C.H., Wagener, F.O.O., 2005. Evolutionary dynamics in markets with many trader types. Journal of Mathematical Economics 42, 7-42.

Brock, W.A., Lakonishok, J., LeBaron, B., 1992. Simple technical trading rules and the stochastic properties of stock returns. Journal of Finance 47, 1731-64.

Broer, H.W., Huitema, G.B., Takens, F., Braaksma, B., 1990. Unfoldings and bifurcations of quasiperiodic tori. Memoirs of the AMS 83. American Mathematical Society: Providence, RI, 1-175.

Bullard, J., 1994. Learning equilibria. Journal of Economic Theory 64, 468-485.

Chenciner, A., 1985a. Bifurcations de points fixes elliptiques. I. Courbes invariantes. IHES-Publications mathématiques 61, 67-127.

Chenciner, A., 1985b. Bifurcations de points fixes elliptiques. II. Orbites periodiques et ensembles de Cantor invariants. Inventiones mathematicae 80, 81-106.

Chenciner, A., 1988. Bifurcations de points fixes elliptiques. III. Orbites périodiques de "petites" périodes et élimination résonnante des couples de courbes invariantes. IHES-Publications mathématiques 66, 5-91.

Chiarella, C., 1992. The dynamics of speculative behaviour. Annals of operations research 37, 101-123. 
Chiarella C., He X.-Z., 2002. Heterogeneous beliefs, risk and learning in a simple asset pricing model. Computational Economics, 19, 95-132.

De Long, J.B., Shleifer, A., Summers, L.H., Waldmann, R.J., 1990. Noise trader risk in financial markets. Journal of Political Economy 98, 703-738.

Engle, R.F., 1982. Autoregressive conditional heteroscedasticity with estimates of the variance of United Kingdom inflation. Econometrica 50, 987-1007.

Evans, G.W., Honkapohja, S., 2001. Learning in macroeconomics. Princeton University Press, Princeton.

Farmer, J.D., Joshi, S., 2002. The price dynamics of common trading strategies. Journal of Economic Behavior and Organization 49, 149-171.

Frankel, J.A., Froot, K.A., 1988. Chartists, fundamentalists and the demand for dollars. Greek Economic Review 10, 49-102.

Friedman, M., 1953. The case of flexible exchange rates. In: Essays in positive economics. University of Chicago Press: Chicago, 157-203.

Gaunersdorfer A., 2000. Endogenous fluctuations in a simple asset pricing model with heterogeneous beliefs. Journal of Economic Dynamics and Control 24, 799-831.

Gaunersdorfer A., 2001. Adaptive belief systems and the volatility of asset prices, Central European Journal of Operations Research 9, 5-30.

Gaunersdorfer A., Hommes C.H., 2007. A nonlinear structural model for volatility clustering. In: Teyssière, G., Kirman, A. (Eds.), Long Memory in Economics. Springer: Berlin/Heidelberg, 265288.

Gaunersdorfer A., Hommes C.H., Wagener, F.O.O., 2000. Bifurcation routes to volatility clustering. CeNDEF Working Paper 00-04, University of Amsterdam and SFB Working Paper 73, University of Vienna and Vienna University of Economics and Business Administration.

http://wwwl.fee.uva.nl/cendef/publications/

http://www.wu-wien.ac.at/am/publications.htm

Gaunersdorfer A., Hommes C.H., Wagener, F.O.O., 2005. Nonlocal onset of instability in an asset pricing model with heterogeneous agents. In: Dumortier F., Broer H., Mawhin J., Vanderbauwhede A., Lunel S.V. (Eds.), EQUADIFF 2003. Proceedings of the international conference on differential equations, Hasselt, Belgium, July 22-26, 2003. Hackensack, NJ: World Scientific. 613-618.

Grandmont, J.-M., 1998. Expectations formation and stability in large socio-economic systems. Econometrica $66,741-81$.

Guckenheimer J., Holmes P., 1986. Nonlinear oscillations, dynamical systems, and bifurcations of vector fields. Springer: New York.

Herman M., 1979. Sur la conjugaison différentiable des difféomorphismes du cercle à des rotations. Publications Mathématiques de l'IHÉS 49, 5-234.

Hommes C.H., 2001. Financial markets as nonlinear adaptive evolutionary systems. Quantitative Finance $1,149-167$. 
Hommes, C.H., 2006. Heterogeneous agent models in economics and finance. In: Tesfatsion, L., Judd, K.L., (Eds.), Handbook of Computational Economics, vol.2: Agent-Based Computational Economics. North-Holland: Amsterdam, 1109-1186.

Hommes, C.H., Sonnemans, J., Tuinstra, J., van de Velden, H., 2005. Coordination of expectations in asset pricing experiments. Review of Financial Studies 18, 955-980.

Kirman, A., 1991. Epidemics of opinion and speculative bubbles in financial markets. In: Taylor, M. (Ed.), Money and financial markets. Macmillan: London, 354-368.

Kuznetsov, Y.A., 1998. Elements of Applied Bifurcation Theory ( $2^{\text {nd }}$ ed.). Springer: New York.

LeBaron, B., 2001. Evolution and time horizons in an agent based stock market. Macroeconomic Dynamics 5, 225-254.

LeBaron, B., 2006. Agent-based computational finance. In: Tesfatsion, L., Judd, K.L., (Eds.), Handbook of Computational Economics, vol.2: Agent-Based Computational Economics. North-Holland: Amsterdam, 1187-1233.

LeBaron, B., Arthur, W.B., Palmer, R., 1999. Time series properties of an artificial stock market. Journal of Economic Dynamics and Control 23, 1487-1516.

Lux, T., 1995. Herd behavior, bubbles and crashes. The Economic Journal 105, 881-896.

Lux, T., 1997. Time variation of second moments from a noise trader/infection Model. Journal of Economic Dynamics and Control 22, 1-38.

Lux, T., Marchesi, M., 1999. Scaling and criticality in a stochastic multi-agent model of a financial market. Nature 397, 498-500.

Lux, T., Marchesi, M., 2000. Volatility clustering in financial markets: a micro-simulation of interacting agents. International Journal of Theoretical and Applied Finance 3, 675-702.

Mandelbrot, B., 1963. The variation of certain speculative prices. Journal of Business 36, 394-419.

Moser, J., 1973. Stable and random motions in dynamical systems. Princeton University Press: Princeton.

Palis, J., Takens, F., 1993. Hyperbolicity and sensitive chaotic dynamics at homoclinic bifurcations. Cambridge University Press: Cambridge.

Pintus, P., Sands, D., Vilder, de R., 2000. On the transition from local regular to global irregular fluctuations. Journal of Economic Dynamics and Control 24, 247-272.

Pomeau, Y., Manneville, P., 1980. Intermittent transition to turbulence in dissipative dynamical systems. Communications in Mathematical Physics 74, 189-197.

Samuelson, L., 1997. Evolutionary games and equilibrium selection. MIT Press: Cambridge.

Saleh, K., 2006. Organising centres in the semi-global analysis of dynamical systems. Ph-D thesis, University of Groningen.

Sargent, T.J., 1993. Bounded Rationality in Macroeconomics. Clarendon Press: Oxford. 
Sargent, T.J., 1999. The Conquest of American Inflation. Princeton University Press: Princeton.

Schönhofer, M., 1999. Chaotic learning equilibria. Journal of Economic Theory 89, 1-20.

Vilder, de R.G., 1996. Complicated dynamics in a two-dimensional overlapping generations model. Journal of Economic Theory 71, 416-442.

Zeeman, E.C., 1974. The unstable behavior of stock exchange. Journal of Mathematical Economics 1, 39-49. 


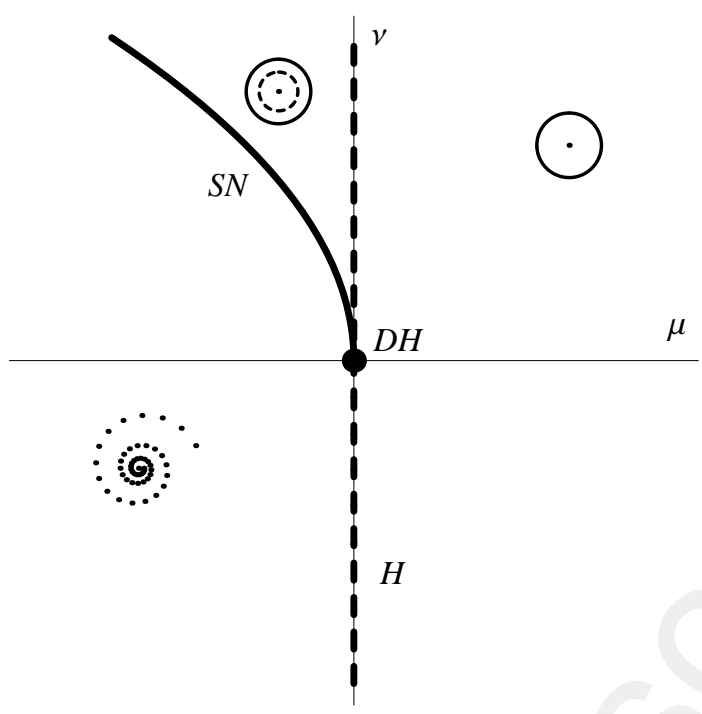

Figure 1: Bifurcation diagram of the Chenciner bifurcation in the $\mu-\nu$-plane. The codimension two bifurcation point DH is in the origin of the coordinate system. The vertical dashed line $H=\{\mu=0\}$ is a curve of Hopf bifurcation values, supercritical on one side of the Chenciner point $(\nu<0)$, subcritical on the other $(\nu>0)$. The solid curve $S N$ denotes a curve of saddlenode bifurcations of invariant circles. The 'volatility clustering region', where a stable steady state and a stable limit cycle coexist, is the region between the curve $S N$ and the positive $\nu$-axes. 


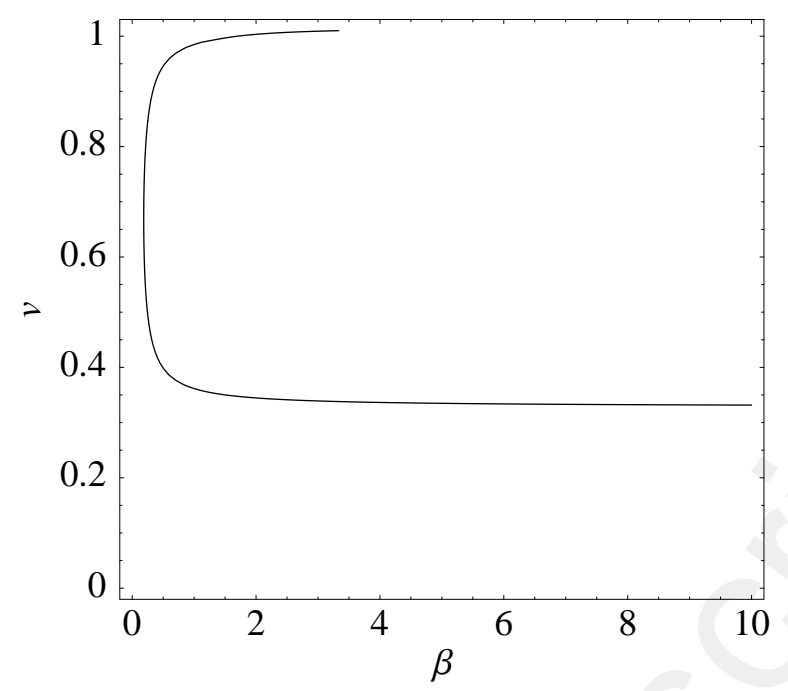

Figure 2: Plot of the curve DH of Chenciner bifurcation points lying within the Hopf bifurcation set $H=\{g=2 R\}$ in the $\beta$-v-diagram, for $\beta \in[0,10], v \in[0,1]$ and $R=1.01$ and $\alpha=1$ fixed. 


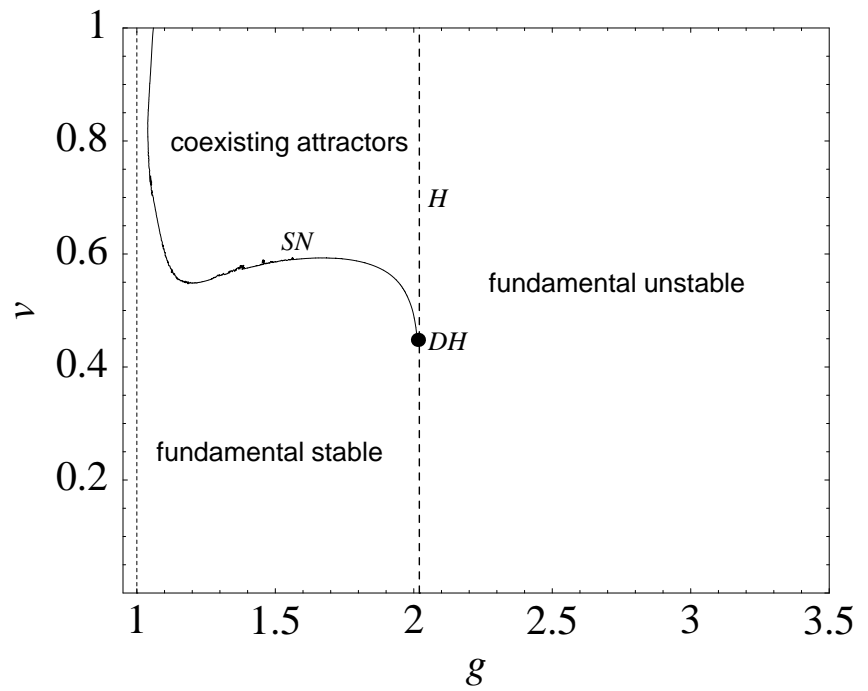

(a) Bifurcation diagram

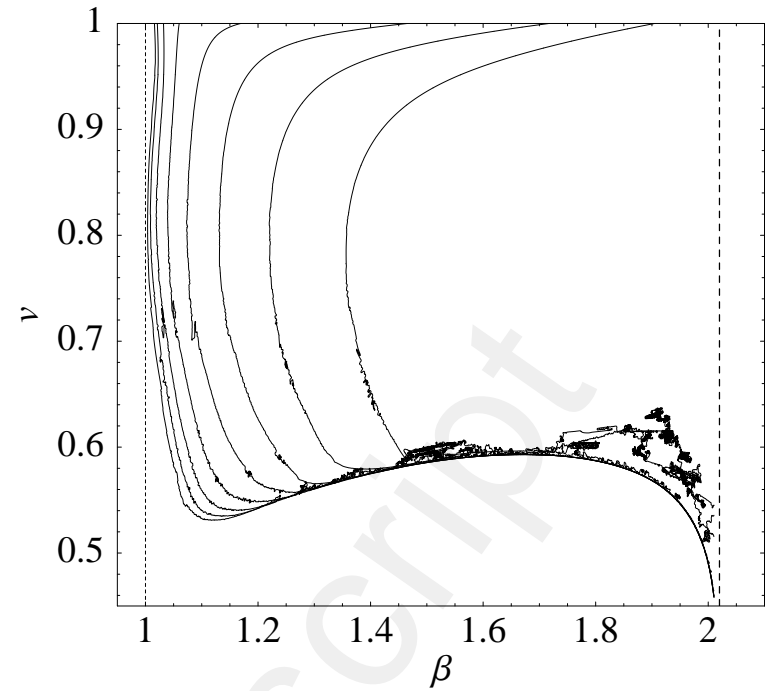

(b) Detail of the bifurcation diagram for various values of $\beta$

Figure 3: Left panel: Bifurcation diagram of the Chenciner bifurcation in the $g$-v-plane for $\beta=$ 1000, $R=1.01$ and $\alpha=10$. At the point labelled $D H(g=2 R=2.02$ and $v \approx 0.45)$ a degenerate Hopf or Chenciner bifurcation occurs. This point lies on the Hopf bifurcation line $\{g=2 R\}$, labeled $H$, and from this point a curve $S N$ emanates corresponding to the saddle-node bifurcation curve of invariant circles. The area between the curves $S N$ and $H$ is the 'volatility clustering region' with a second attractor coexisting with the stable fundamental steady state. Right panel: The curve $S N$, corresponding to the saddle-node bifurcation of the invariant circle, for different values of the intensity of choice $\beta=10^{i / 2}, i=2, \ldots, 8$. The curve $S N$ moves to the left and approaches the vertical line $g=1$, as the intensity of choice increases from $\beta=10$ to $\beta=10,000$. 


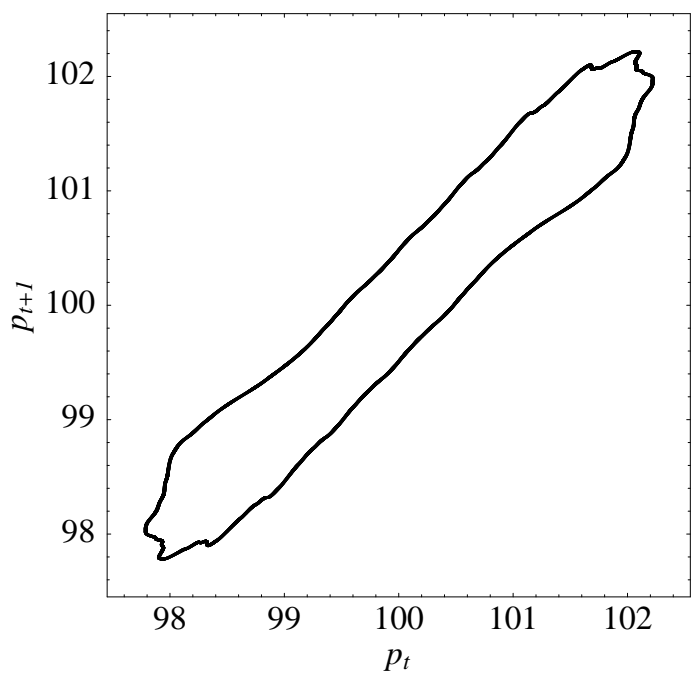

(a) $g=1.6$

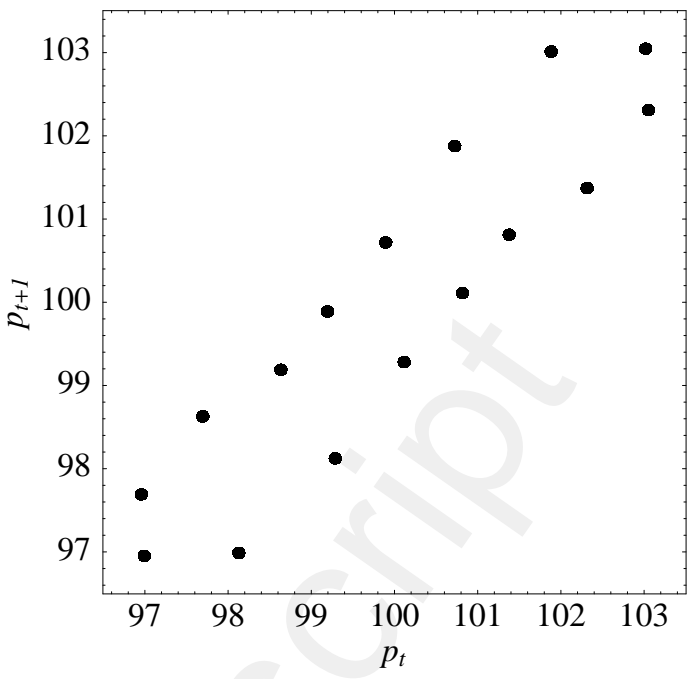

(b) $g=1.71$

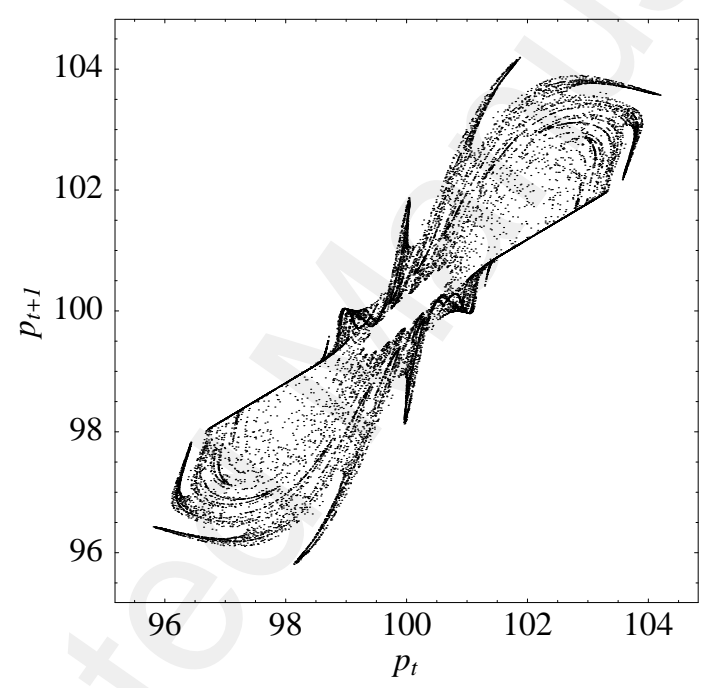

(c) $g=2$

Figure 4: Projections of a quasi-periodic, a periodic and a chaotic attractor on the $p_{t}-p_{t+1^{-}}$ plane. Not drawn is the stable fundamental steady state at $p^{*}=100$. Parameters are $\beta=4$, $v=0.6, R=1.01, \alpha=10$, and, from left to right, $g=1.60, g=1.71$, and $g=2.00$. These parameter values lie in the 'volatility clustering region' where two attractors coexist. The fixed point undergoes a subcritical Hopf bifurcation at $g=2.02$. In the right figure the unstable invariant circle can be seen as the inner boundary of the strange attractor. 


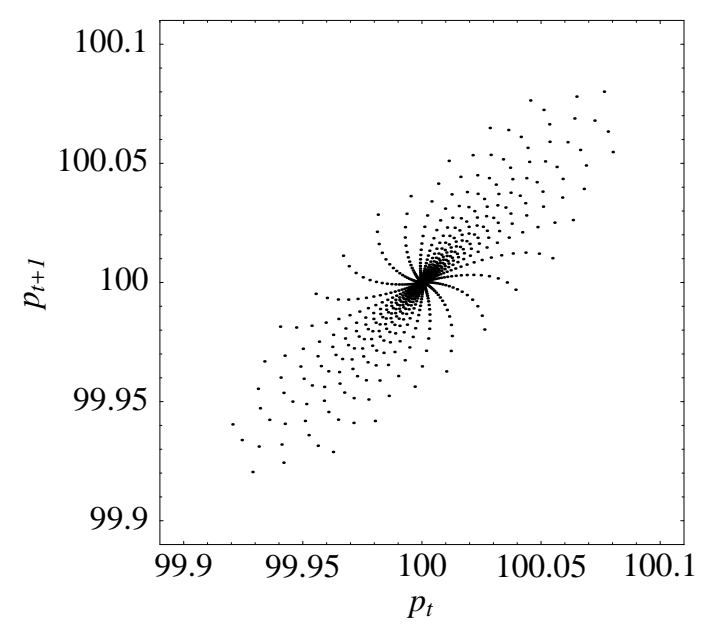

(a) $\mathrm{g}=2$

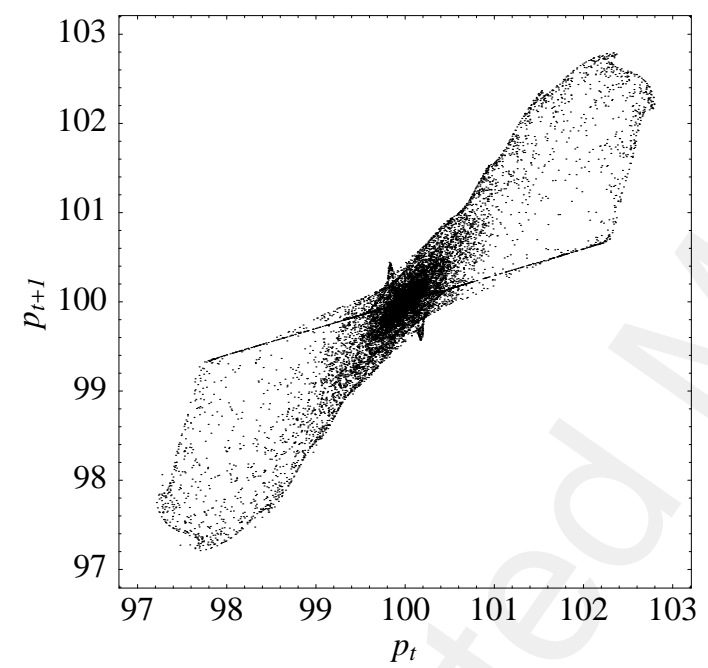

(c) $\mathrm{g}=2.4$

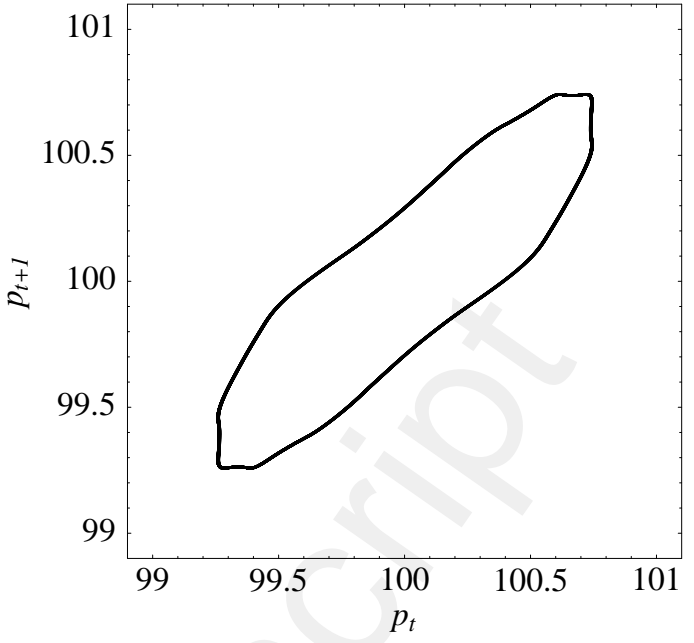

(b) $\mathrm{g}=2.09$

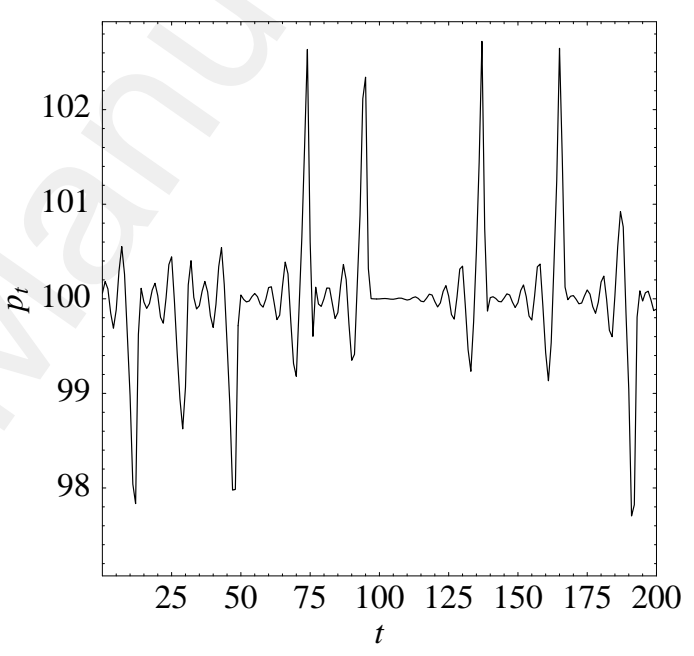

(d) $\mathrm{g}=2.4$, time series

Figure 5: Projection of the attractors on the $p_{t}-p_{t+1}$-plane. Parameters are $\beta=4, v=0.3$, $R=1.01, \alpha=10$ and $g=2(a), g=2.09(b)$ and $g=2.4(c)$. The fixed point is attracting in the top left picture: points spiraling towards it are shown. It undergoes a supercritical Hopf bifurcation at $g=2.02$, has a quasiperiodic attractor for $g=2.09$ and a strange attractor for $g=2.4$, with corresponding time series for the strange attractor in the plot $(d)$ showing intermittent chaos. 


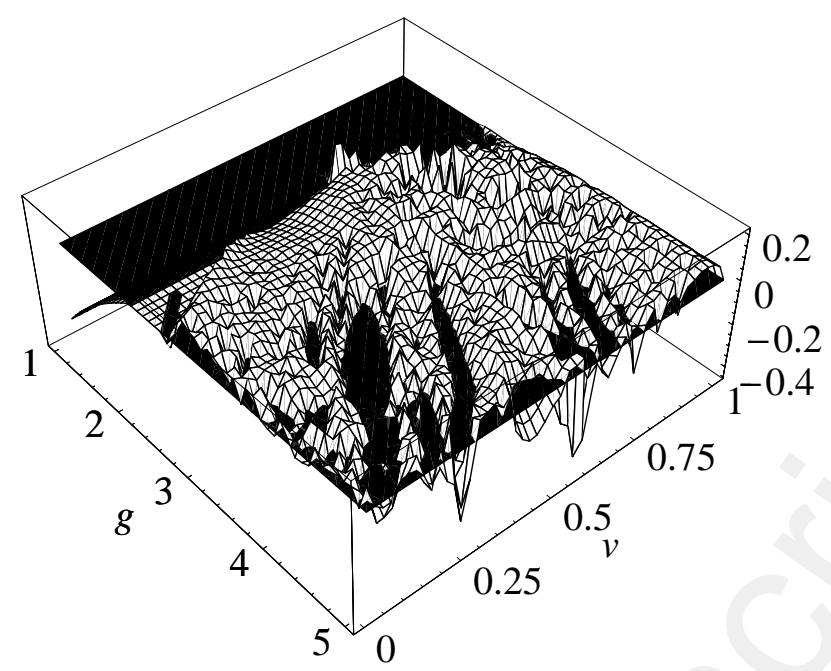

(a) Lyapunov exponents

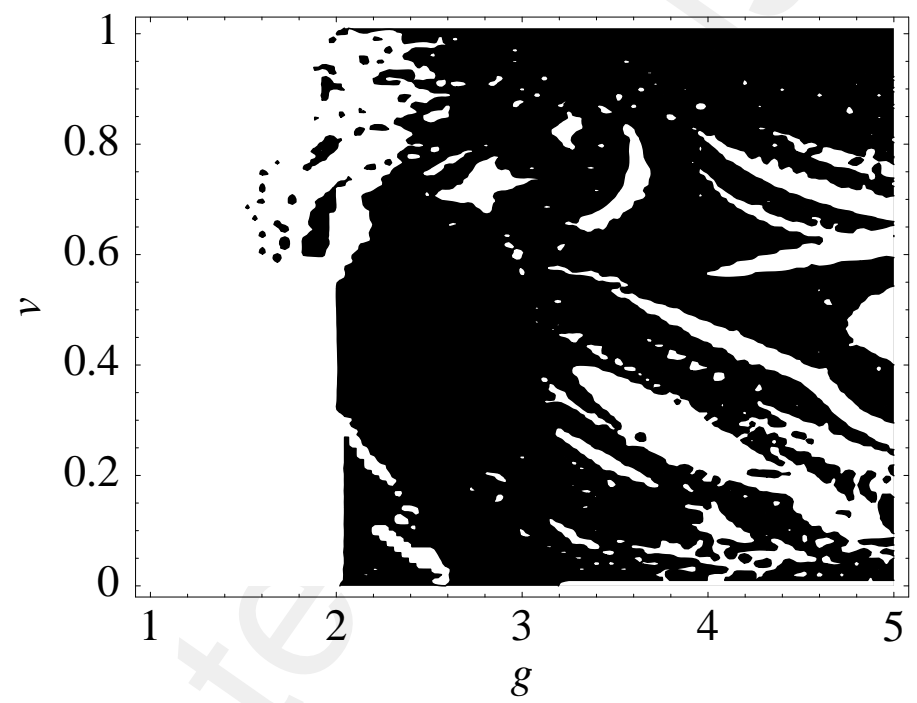

(b) Parameter region of positive Lyapunov exponents

Figure 6: Lyapunov exponents for $g \in[1,5], v \in[0,1], \beta=3, R=1.01, \alpha=10$ : on the left, the magnitude of the largest Lyapunov exponent is plotted along the z-axis. In the right picture, for points in the grey area, largest Lyapunov exponents are positive; for those parameters, there is a chaotic attractor. 


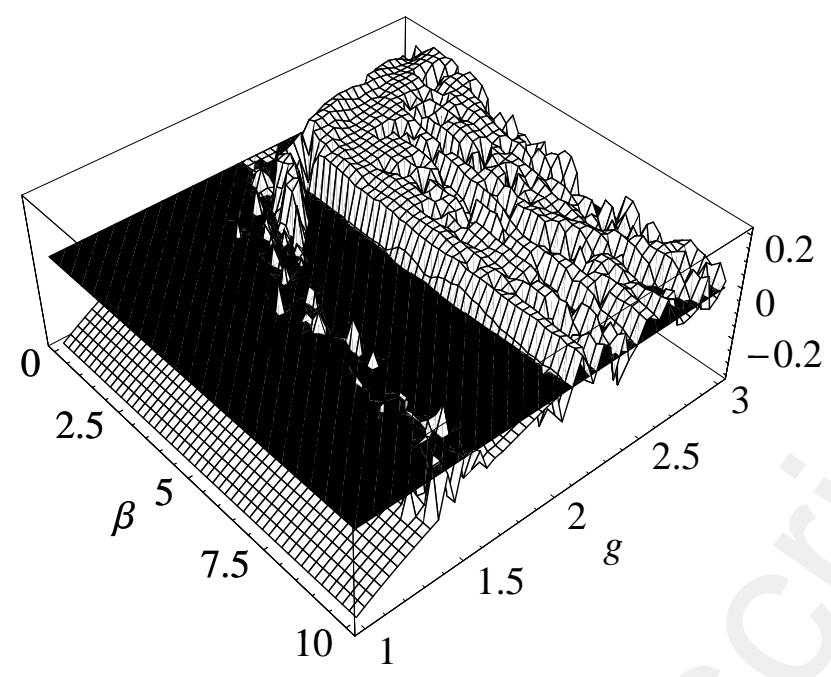

(a) Lyapunov exponents

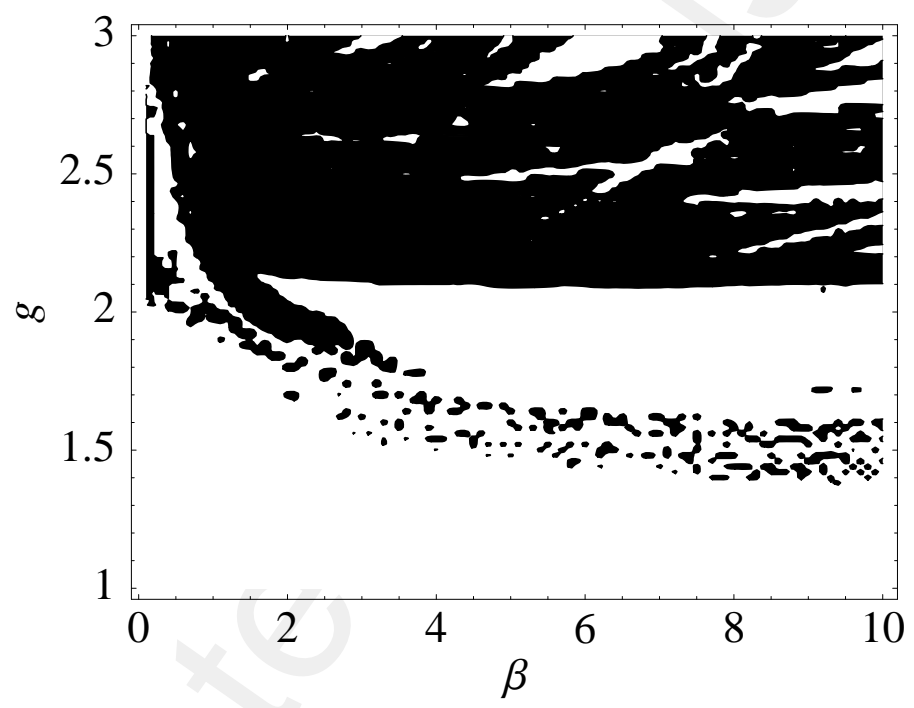

(b) Parameter region of positive Lyapunov exponents

Figure 7: Lyapunov exponents for $\beta \in[0,10], g \in[1,3], v=0.6, R=1.01, \alpha=10$. Legend as in figure 6 . 


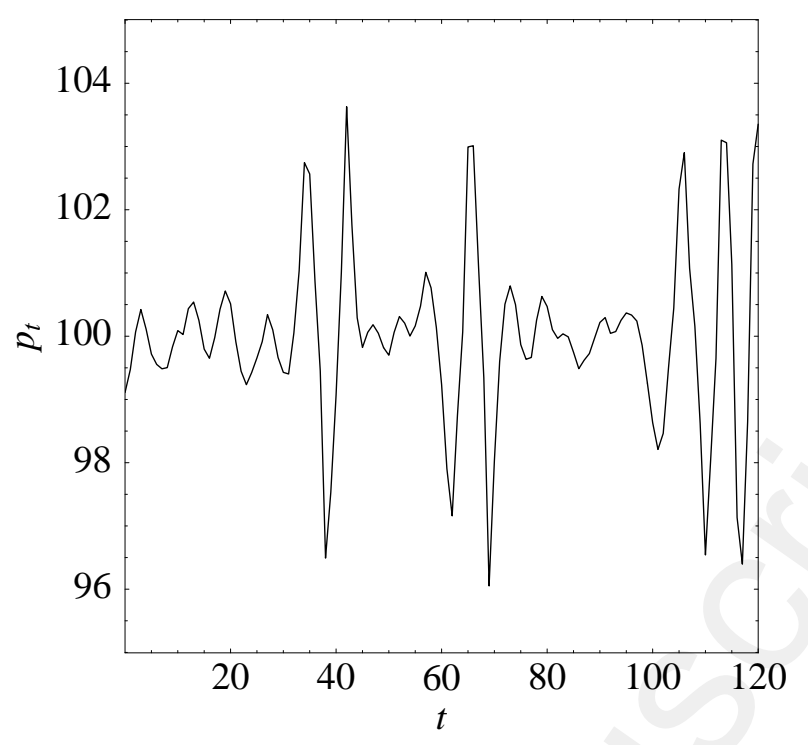

Figure 8: Time series of prices for the model with fundamentalists and chartists together with a small fraction of noise traders. Parameters are such that a stable fundamental steady state and a strange attractor coexist (see figure $4 c$ ). Due to the noise traders, price fluctuations switch irregularly between a low volatility phase, with prices close to the fundamental value, and a high volatility phase, with prices exhibiting temporary bubbles. 
Generic

Page 36 of 36 